\title{
Whole genome sequencing of a novel, dichloromethane- fermenting Peptococcaceae from an enrichment culture
}

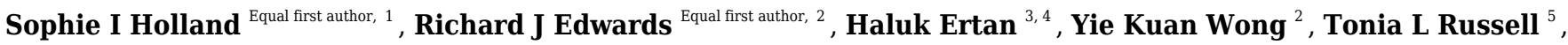 \\ Nandan P Deshpande ${ }^{2}$, Michael Manefield ${ }^{1,4}$, Matthew J Lee ${ }^{\text {Corresp. } 1}$ \\ ${ }^{1}$ School of Civil and Environmental Engineering, University of New South Wales, Sydney, New South Wales, Australia \\ 2 School of Biotechnology and Biomolecular Sciences, University of New South Wales, Sydney, New South Wales, Australia \\ 3 Department of Molecular Biology and Genetics, Istanbul University, Istanbul, Turkey \\ 4 School of Chemical Engineering, University of New South Wales, Sydney, New South Wales, Australia \\ 5 Ramaciotti Centre for Genomics, University of New South Wales, Sydney, New South Wales, Australia \\ Corresponding Author: Matthew J Lee \\ Email address: mattlee@unsw.edu.au
}

Bacteria capable of dechlorinating the toxic environmental contaminant dichloromethane (DCM, $\mathrm{CH}_{2} \mathrm{Cl}_{2}$ ) are of great interest for potential bioremediation applications. A novel, strictly anaerobic, DCM-fermenting bacterium, "DCMF", was enriched from organochlorinecontaminated groundwater near Botany Bay, Australia. The enrichment culture was maintained in minimal, mineral salt medium amended with dichloromethane as the sole energy source. PacBio whole genome SMRT ${ }^{\mathrm{TM}}$ sequencing of DCMF allowed de novo, gapfree assembly despite the presence of cohabiting organisms in the culture. Illumina sequencing reads were utilised to correct minor indels. The single, circularised $6.44 \mathrm{Mb}$ chromosome was annotated with the IMG pipeline and contains 5,773 predicted proteincoding genes. Based on 16S rRNA gene and predicted proteome phylogeny, the organism appears to be a novel member of the Peptococcaceae family. The DCMF genome is large in comparison to known DCM-fermenting bacteria. It includes an abundance of methyltransferases, which may provide clues to the basis of its DCM metabolism, as well as potential to metabolise additional methylated substrates such as quaternary amines. Full annotation has been provided in a custom genome browser and search tool, in addition to multiple sequence alignments and phylogenetic trees for every predicted protein, available at http://www.slimsuite.unsw.edu.au/research/dcmf/. 


\section{Whole genome sequencing of a novel,}

2 dichloromethane-fermenting Peptococcaceae from 3 an enrichment culture

4 Sophie I Holland $^{\mathrm{a}} \uparrow$, Richard J Edwards ${ }^{\mathrm{b}} \uparrow$, Haluk Ertan ${ }^{\mathrm{c}, \mathrm{d}}$, Yie Kuan Wong ${ }^{\mathrm{b}}$, Tonia Russelle,

5 Nandan Deshpande ${ }^{\mathrm{b}}$, Michael Manefielda,d, Matthew Lee ${ }^{\mathrm{a}^{*}}$

6

$7 \quad \dagger$ These authors contributed equally to this work.

9 aSchool of Civil and Environmental Engineering, University of New South Wales, Sydney,

10 NSW, Australia

11 bSchool of Biotechnology and Biomolecular Sciences, University of New South Wales, Sydney,

12 NSW, Australia

13 'Department of Molecular Biology and Genetics, Istanbul University, Istanbul, Turkey

14 dSchool of Chemical Engineering, University of New South Wales, Sydney, NSW, Australia

eRamaciotti Centre for Genomics, University of New South Wales, Sydney, NSW, Australia

17 Running title: Genome sequence of a novel DCM fermenter.

*Corresponding Author:

20 Matthew Lee

21 Email address: mattlee@unsw.edu.au 


\section{Abstract}

24 Bacteria capable of dechlorinating the toxic environmental contaminant dichloromethane (DCM,

25

26

27

28

29

30

31

32

33

34

35

36

37

38

$\mathrm{CH}_{2} \mathrm{Cl}_{2}$ ) are of great interest for potential bioremediation applications. A novel, strictly anaerobic, DCM-fermenting bacterium, "DCMF”, was enriched from organochlorinecontaminated groundwater near Botany Bay, Australia. The enrichment culture was maintained in minimal, mineral salt medium amended with dichloromethane as the sole energy source. PacBio whole genome SMRT ${ }^{\mathrm{TM}}$ sequencing of DCMF allowed de novo, gap-free assembly despite the presence of cohabiting organisms in the culture. Illumina sequencing reads were utilised to correct minor indels. The single, circularised $6.44 \mathrm{Mb}$ chromosome was annotated with the IMG pipeline and contains 5,773 predicted protein-coding genes. Based on 16S rRNA gene and predicted proteome phylogeny, the organism appears to be a novel member of the Peptococcaceae family. The DCMF genome is large in comparison to known DCM-fermenting bacteria. It includes an abundance of methyltransferases, which may provide clues to the basis of its DCM metabolism, as well as potential to metabolise additional methylated substrates such as quaternary amines. Full annotation has been provided in a custom genome browser and search tool, in addition to multiple sequence alignments and phylogenetic trees for every predicted protein, available at http://www.slimsuite.unsw.edu.au/research/dcmf/.

\section{Introduction}

Dichloromethane (DCM, $\mathrm{CH}_{2} \mathrm{Cl}_{2}$ ) is a toxic environmental contaminant. Approximately $70 \%$ of all DCM worldwide is of anthropogenic origin, due to its extensive use in industry as a solvent and aerosol propellant (Gribble, 2009; Marshall \& Pottenger, 2016). It is currently present at $30 \%$ of Superfund National Priority List sites within the United States and its territories (U.S. National Library of Medicine, 2019), and global capacity for DCM continues to steadily increase (Marshall \& Pottenger, 2016). As well as being harmful to human health (Agency for Toxic Substances and Disease Registry, 2000), DCM has recently been recognised as a potent greenhouse gas (Hossaini et al., 2017).

DCM in groundwater can be transformed by both aerobic and anaerobic bacteria, although the former has been more comprehensively characterized (Leisinger \& Braus-Stromeyer, 1995). Aerobic DCM metabolism is found in facultative methylotrophs, which use a DCM dehydrogenase from the glutathione S-transferase family to catalyse dehalogenation (Leisinger \& Braus-Stromeyer, 1995). Anaerobically, DCM can be transformed under denitrifying conditions (Melendez, Roman \& Smith, 1993; Freedman, Smith \& Noguera, 1997), or co-metabolically under methanogenic conditions (Freedman \& Gossett, 1991; Stromeyer et al., 1991). To date, however, only two DCM-fermenting bacteria have been described and sequenced: Dehalobacterium formicoaceticum (Mägli, Wendt \& Leisinger, 1996; Chen et al., 2017) and 'Candidatus Dichloromethanomonas elyunquensis' (Kleindienst et al., 2016, 2017). Of these, only the former has been isolated (Mägli, Wendt \& Leisinger, 1996).

Both species are acetogenic and are thought to metabolise DCM via incorporation of the methyl group into the Wood-Ljungdahl pathway (reviewed in Ragsdale and Pierce, 2008), although the 
62 precise mechanism of dechlorination has thus far eluded description (Mägli, Wendt \& Leisinger, 63 1996; Kleindienst et al., 2017). The Wood-Ljungdahl pathway is an ancient metabolism that is 64 present in acetogenic bacteria and methanogenic archaea, and links carbon fixation with ATP

65

66

67

68

69

70

71

72

73

74

75

76

77

78

79

80

81

82

83

84

85

86

87

88

89

90

91

92

93

94

95

96

97

98

generation (Fuchs, 2011; Poehlein et al., 2012). It is thought that an as-yet unidentified methyltransferase (D. formicoaceticum) possibly in concert with a reductive dehalogenase (' $\mathrm{Ca}$. Dichloromethanomonas elyunquensis') is responsible for mediating the transformation of DCM into methyl- or methylene-tetrahydrofolate (Kleindienst et al., 2019).

In order to investigate possible pathways for DCM transformation within the novel organism described in this paper, we determined that a detailed and accurate genome annotation was necessary. As it can be difficult to assemble a high quality genome from a mixed culture, we sought to overcome these challenges with a thorough genome sequencing and assembly strategy. We report the whole genome sequencing and assembly of a novel, DCM-fermenting bacterium, herein referred to as DCMF. The organism exists in an enrichment culture, "DFE" (DCMfermenting enrichment), derived from a previously reported methanogenic, DCM-dechlorinating consortium, DCMD (Lee et al., 2012). DCMD was dominated by a Dehalobacter species whose growth was linked to DCM metabolism. The enrichment process reported here led to a shift from this Dehalobacter species to DCMF as the dominant member of the community. From a bioremediation perspective, DCMF is an important addition to the limited group of organisms able to utilize the common environmental pollutant DCM.

\section{Materials \& Methods}

Inoculum origin

The original inoculum was obtained from sediment drilled from $5 \mathrm{~m}$ beneath the surface of an organochlorine-contaminated coastal sand bed aquifer (Botany Sands aquifer), latitude $33^{\circ} 57^{\prime} 27.6^{\prime} \mathrm{S}$, longitude $151^{\circ} 12^{\prime} 60.0^{\prime} \mathrm{E}$. The initial, methanogenic enrichment culture using DCM as the sole energy source was reported previously (Lee et al., 2012).

Culture media

Cultures were grown in anaerobic minimal mineral salts medium that comprised $\left(\mathrm{g} \mathrm{l}^{-1}\right)$ :

$\mathrm{CaCl}_{2} .2 \mathrm{H}_{2} \mathrm{O}(0.1), \mathrm{KCl}(0.1), \mathrm{MgCl}_{2} \cdot 6 \mathrm{H}_{2} \mathrm{O}(0.1), \mathrm{NaHCO}_{3}(2.5), \mathrm{NH}_{4} \mathrm{Cl}(1.5), \mathrm{NaH}_{2} \mathrm{PO}_{4}(0.6), 1$ $\mathrm{ml}$ of trace element solution $\mathrm{A}(1000 \times), 1 \mathrm{ml}$ of trace element solution $\mathrm{B}(1000 \times), 1 \mathrm{ml}$ of vitamin solution $(1000 \times), 10 \mathrm{ml}^{\circ} 5 \mathrm{~g} \mathrm{l}^{-1}$ fermented yeast extract (FYE; 100 $\times$ ), and resazurin $0.25 \mathrm{mg} \mathrm{l}^{-1}$. Trace element solutions A and B were prepared as described previously (Wolin, Wolin \& Wolfe, 1963), as was the vitamin solution (Adrian et al., 1998). Medium was sparged with $\mathrm{N}_{2}$ during preparation and the $\mathrm{pH}$ was adjusted to $6.8-7.0$ by a final purge with $\mathrm{N}_{2} / \mathrm{CO}_{2}$ (4:1). Aliquots were dispensed into glass serum bottles that were crimp sealed with Teflon faced rubber septa (13 $\mathrm{mm}$ diameter, Wheaton) before the medium was chemically reduced with sodium sulphide $(0.2 \mathrm{mM})$. DCM $(1 \mathrm{mM})$ was supplied as the sole electron source via a glass syringe. Methanogenic Archaea present in the early enrichment culture were inhibited with 2-

Peer) reviewing PDF | (2019:05:37241:2:0:NEW 23 Aug 2019) 
99 bromoethanosulfonate (BES, $0.2 \mathrm{mM}$ ) for two generations. All cultures were incubated statically 100 at $30^{\circ} \mathrm{C}$ in the dark.

101 Preparation of spent media as a co-factor solution

102 A stock FYE solution was prepared by inoculating anoxic yeast extract $\left(5 \mathrm{~g} \mathrm{l}^{-1}\right)$ in defined 103 minimal mineral salts medium (described above, excluding DCM) with the DFE culture. The 104 culture was incubated for one week at $30^{\circ} \mathrm{C}$ before being filter-sterilised. The filtered, spent 105 media was re-inoculated with DFE and incubated for a further week, to ensure that growth was 106 no longer possible on FYE (i.e. that it had been energetically exhausted). The spent media was 107 then filter-sterilised again before use.

108 Analytical methods

109 DCM and methane were quantified on a GS-Q column $(30 \mathrm{~m} \times 0.32 \mathrm{~mm}$; Agilent Technologies $)$

110 using a Shimadzu GC-2010 gas chromatograph with flame ionisation detector (GC-FID).

111 Headspace samples $(100 \mu \mathrm{l})$ were withdrawn directly from culture flasks using a lockable, gas-

112 tight syringe and injected manually. The oven was initially $150^{\circ} \mathrm{C}$, then raised by $30^{\circ} \mathrm{C} \mathrm{min}^{-1}$ to

$113250^{\circ} \mathrm{C}$. The inlet temperature was $250^{\circ} \mathrm{C}$, split ratio $1: 10$, FID temperature $250^{\circ} \mathrm{C}$. A minimum

114 three-point calibration curve was used. DCM concentrations are reported as the nominal

115 concentration in each serum bottle, calculated from the headspace concentration using the

116 Henry's Law dimensionless solubility constant $\left(H^{c c}=0.107\right.$ at $\left.30^{\circ} \mathrm{C}\right)$, as per the OSWER method

117 (US EPA, 2001).

118 Genomic DNA extraction

119 Genomic DNA was extracted as previously described (Urakawa, Martens-Habbena \& Stahl, 120 2010). Briefly, cells were lysed with lysis buffer and bead-beating, before DNA was extracted 121 with phenol-chloroform-isoamyl, precipitated using isopropanol, and resuspended in molecular 122 grade water. The nucleic acid concentration was quantified using a Qubit instrument and assay as 123 per the manufacturer's instructions (Life Technologies).

124 Community analysis

125 Throughout the initial transfers and serial dilutions of the enrichment culture, the community was 126 monitored via denaturing gradient gel electrophoresis (DGGE). DNA was amplified with primers

127 GC338F and 530R (Table S1). DGGE was performed with a DCode mutation detection system 128 (Bio-Rad) and a Cipher Electrophoresis system (CBS Scientific Company Inc) in a $1 \times$ TAE 129 buffer at $\mathrm{pH}$ 7.5. PCR products were loaded onto a $10 \%(\mathrm{v} / \mathrm{v})$ acrylamide gel with a $30-60 \%$ 130 gradient of urea and deionised formamide before electrophoresis at $60^{\circ} \mathrm{C}, 75 \mathrm{~V}$ for $16.5 \mathrm{~h}$. Gels 131 were stained with SYBR Gold (Invitrogen ${ }^{\mathrm{TM}}$, Life Technologies) in 1× TAE buffer for $10 \mathrm{~min}$, 132 prior to visualisation on a Gel Doc XR (Bio-Rad). Bands of interest were excised, DNA eluted 133 from them in molecular grade water and re-amplified using the 338F primer (Table S1). PCR 134 products were cleaned with a Clean and Concentrate-25 kit (Zymo Research).

135 To confirm the absence of an archaeal population following amendment of the enrichment 136 culture with BES, archaeal specific primers Arc340F and Arc1000R (Table S1) were used for 137 PCR on a T100 ${ }^{\mathrm{TM}}$ thermal cycler (Bio-Rad). 
138 Quantitative PCR of the Dehalobacter spp. 16S rRNA gene was carried out on a CFX96 thermal

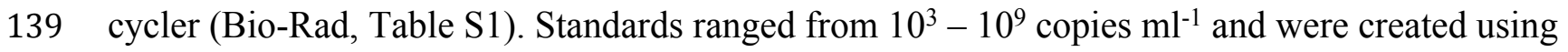
140 serial 10-fold dilutions of a plasmid carrying the cloned gene, constructed with TOPO TA

141 Cloning Kit (Life Technologies).

142 Illumina genome sequencing

143 DNA was prepared with the Nextera XT library prep kit (Illumina). Sequencing was carried out 144 on an Illumina MiSeq with a v2 500-cycle kit ( $2 \times 250$ bp run) at the Ramaciotti Centre for

145 Genomics (UNSW Sydney, Australia). Three MS110-2 libraries were used for the run. Library 146 size ranged from $200-3000 \mathrm{bp}$, with an average of $955 \mathrm{bp}$. Raw reads were trimmed and filtered 147 with SolexaQA (DynamicTrim.pl and LengthSort.pl) (Cox, Peterson \& Biggs, 2010). Raw reads 148 were submitted to the NCBI Sequence Read Archive with the identifier SRR5179547.

149 Pacific Biosciences SMRT sequencing

150 A MagAttract HMW DNA kit (Qiagen) was used to extract high-molecular weight genomic

151 DNA, followed by purification using AMPure PB beads (Beckman Coulter). DNA concentration 152 and purity were checked by Qubit and NanoDrop instruments, respectively. A 0.75\% Pippin 153 Pulse gel (Sage Science) was performed by the Ramaciotti Centre for Genomics (UNSW 154 Sydney, Australia) to further verify integrity. A SMRTbell library was prepared with the PacBio $15520 \mathrm{~kb}$ template protocol excluding shearing (Pacific BioSciences). Additional damage repair was 156 carried out following minimum $4 \mathrm{~kb}$ size selection using Sage Science BluePippin.

157 Whole genome sequencing was performed on the PacBio RS II (Pacific Biosciences), employing 158 P6 C4 chemistry with 240 min movie lengths. DNA was initially sequenced using two Single 159 Molecule Real Time ${ }^{\mathrm{TM}}$ (SMRT) cells. A third SMRT ${ }^{\mathrm{TM}}$ cell was added to compensate for low 160 quality data from the first two, due to degraded DNA yield from the sample. The SMRTbell 161 library for this cell was prepared with the PacBio $10 \mathrm{~kb}$ template protocol, without size selection, 162 and a lower input (3,624 ng) of DNA was used. In total, the three SMRT cells yielded 463,878 163 subreads from 169,180 ZMW, with a combined length of 1,712,588,985 bp. Reads were 164 submitted to the NCBI Sequence Read Archive with the identifier SRR5179548.

165 Genome assembly and annotation

166 PacBio subreads were assembled using HGAP3 (Chin et al., 2013) as implemented in SMRT 167 Portal. In-house software, SMRTSCAPE (SMRT Subread Coverage \& Assembly Parameter 168 Estimator; http://rest.slimsuite.unsw.edu.au/smrtscape) was used to predict optimal HGAP 169 settings for several different assemblies with different predicted genome size and minimum 170 correction depths (Table S2). The assembly with the greatest depth of coverage used for seed 171 read error correction that still yielded a full-length $(6.44 \mathrm{Mb})$ intact chromosome was selected for 172 the draft genome. This corresponded to: min read length $4,010 \mathrm{bp}$; min seed read length 8,003 $173 \mathrm{bp}$; min read quality 0.86 ; min $10 \times$ correction coverage. The genome was corrected with Quiver 174 (Chin et al., 2013) using all subreads and circularised by identifying and trimming overlapping 175 ends, then annotated in-house using Prokka (Seemann, 2014). 
176 Based on draft annotation, the genome was re-circularised to have its break-point in the

177 intergenic region between the 3' of two hypothetical genes, and then subjected to a second round

178 of Quiver correction to make sure the manually joined region was of high quality. Filtered

179 Illumina reads were mapped onto the Quiver-corrected genome using BWA-MEM v0.7.9a (Li,

180 2013) and possible errors were identified with Pilon (Walker et al., 2014). Manual curation was

181 then performed to check any discrepancies between the PacBio and Illumina data and correct

182 small indels. Raw PacBio reads were mapped onto the completed genome with BLASR

183 (Chaisson \& Tesler, 2012). The corrected genome was re-annotated with Prokka and uploaded to

184 the Integrated Microbial Genomes and Microbiomes (IMG/M) system of the Joint Genome

185 Institute (JGI) for independent annotation (Chen et al., 2019).

186 Twenty-eight fragmented pairs of genes were subject to additional manual curation and

187 correction where a pyrrolysine or selenocysteine residue had been erroneously translated as a

188 stop codon (Table S3). The JGI annotation was publically updated to reflect these manual

189 annotations, and this annotation was used for all genomic analyses. The genome has

190 subsequently been re-annotated by NCBI. An overview of the genome assembly and annotation

191 pipeline is provided in Figure 1.

192 16S rRNA gene identification and phylogeny

193 The DCMF 16S rRNA gene consensus sequence was searched against the NCBI prokaryotic 16S

194 rRNA BLAST database as well as the 16S rRNA gene sequences of the two other known DCM-

195 fermenting bacteria (absent from that database), D. formicoaceticum strain DMC (NCBI locus

196 tags CEQ75_RS05455, CEQ75_RS05490, CEQ75_RS13675, CEQ75_RS13970,

197 CEQ75_RS17045) and ' $C a$. Dichloromethanomonas elyunquensis' strain RM (KU341776.1).

198 The closest phylogenetic relatives and an outgroup, Moorella perchloratireducens strain An10

199 (NR_125518.1), were aligned with MAFFT program v.7 (Kuraku et al., 2013) and a neighbour-

200 joining tree constructed with 1000 bootstraps resampling a 200PAM/k $=2$ scoring matrix using

201 1,365 nucleotides. This was performed using Archaeopteryx (Han \& Zmasek, 2009), as well as

202 manual curation. In addition, DCMF 16S rRNA gene sequences were mapped to taxa using the

203 SILVA Alignment, Classification and Tree (ACT) Service (Pruesse, Peplies \& Glöckner, 2012)

204 with default values.

205 In order to identify any non-DCMF 16S rRNA genes from the PacBio sequencing data, all

206 contigs from all of the attempted assemblies were combined into a single file. DCMF contigs

207 were identified and removed and the remaining contigs reduced to a set that were non-redundant

208 at the level of $99 \%$ global sequence identity for the shorter contig, using GABLAM (Davey,

209 Shields \& Edwards, 2006). Where redundancy was identified, the longer contig was retained. In

210 total, 20,201 contigs were reduced to 1,538 non-redundant non-DCMF contigs, hereon referred

211 to as "NR contaminants". Cohabitant bacteria 16S rRNA gene sequences were identified from

212 NR contaminants using barrnap v0.9 (implementing HMMer v3.2.1 and bedtools v2.27.1).

213 Sequences were mapped to taxa using the SILVA Alignment, Classification and Tree (ACT)

214 Service (Pruesse, Peplies \& Glöckner, 2012) with default values. 
215 High throughput phylogenetic analysis of predicted proteome

216 JGI-annotated DCMF proteins were further annotated via high-throughput homology searching,

217 multiple sequence alignment and molecular phylogenetics using HAQESAC v1.10.2 (Edwards et

218 al., 2007). BLAST + v2.6.0 blastp (Camacho et al., 2009) was used to search each protein against

219 three protein datasets: (1) all bacterial proteins in the UniProt Knowledgebase (The UniProt

220 Consortium, 2017) (downloaded 2017-02-06); (2) the predicted DCMF proteome; (3) the nine

221 NCBI proteomes available for closely related bacteria identified from 16S rRNA gene analysis:

222 D. formicoaceticum (GCF_002224645.1), Desulfosporosinus acididurans (GCF_001029285.1),

223 Desulfosporosinus acidiphilus (GCF_000255115.2), Desulfosporosinus orientis

224 (GCF_000235605.1), Desulfosporosinus hippei (GCF_900100785.1), Desulfosporosinus lacus

225 (GCF_900129935.1), Desulfitobacterium metallireducens (GCF_000231405.2),

226 Desulfitobacterium hafniense (GCF_000021925.1), Dehalobacter restrictus

227 (GCF_000512895.1). The top 50 blastp results for each dataset were combined and up to 60

228 homologues meeting the HAQESAC default filtering criteria were aligned with Clustal Omega

229 v1.2.2 (Sievers \& Higgins, 2017). Neighbour-joining phylogenetic trees (1000 bootstraps) were

230 inferred using ClustalW v2.1 and midpoint-rooted using HAQESAC. Paralogous subfamilies

231 arising from gene duplications were identified as nodes where the two ancestral clades each had

232 at least two different species and shared at least one of those species. Multiple sequences from

233 the same species within one of these paralogous subfamilies were identified as "in-paralogues"

234 (lineage-specific duplications) or possible sequence variants. DCMF in-paralogues were kept.

235 Possible in-paralogues or sequence variants from other species were restricted to the single

236 closest homologue to the DCMF query. NCBI annotated proteins were subsequently subjected to

237 the same pipeline with the addition of the JGI predicted proteome to the search database.

238 Putative taxonomic assignments for each JGI protein were made using an in-house tool,

239 TaxaMap (http://rest.slimsuite.unsw.edu.au/taxamap). TaxaMap identifies the smallest clade to

240 which the query DCMF protein can be confidently assigned by stepping ancestrally through the

241 tree until it reaches a branch with a bootstrap support of at least $50 \%$ and at least one non-DCMF

242 protein. If the root is reached without meeting these requirements, the full HAQESAC tree was

243 used. Once the clade has been identified, all Uniprot species codes for that clade are extracted as

244 putative taxonomic assignments. These are mapped onto parent species, genus, family, order,

245 class and phylum classifications using UniProt Knowledgebase taxonomy. At each taxonomic

246 level, the taxa list is reduced to be non-redundant and each taxon contributes equally, to reduce

247

248 sampling biases. Where a species code could only be mapped to a higher taxonomic level, it was

249 designated as an unknown taxon associated with that higher level, e.g. "Firmicutes fam." would indicate an unknown family within the phylum Firmicutes. Where no non-DCMF homologues

250

251

252

253

254 were found, a protein was assigned "None". TaxaMap Assignments were made for each protein individually and then combined using two strategies: (1) Unweighted; (2) Bootstrap weighted. The unweighted assignment simply adds up the number of proteins assigned to a particular taxon. Where a protein is assigned to multiple taxa, each is given an equal proportion of that protein, e.g. if a protein mapped ambiguously to five taxa, each would receive 0.2 for that 
255

256

257

258

259

260

261

262

263

264

265

266

267

268

269

270

271

272

273

274

275

276

277

278

279

280

281

282

283

284

285

286

287

288

289

290

291

292

protein. Any taxa with a combined score below 1.0 across all proteins was excluded, and scores recalculated iteratively. For the weighted score, counts were multiplied by the percentage bootstrap support for the clade, e.g. if a protein was assigned to two taxa and the bootstrap support for the clade was $80 \%$, each taxon would receive a score of $0.4(=0.5 \times 0.8)$.

A subset of eight core house-keeping genes and 47 ribosomal proteins (Table S4) was subject to re-analysis using Maximum Likelihood trees (1000 bootstraps) inferred by IQTree v1.6.1 using ModelFinder (Nguyen et al., 2015; Kalyaanamoorthy et al., 2017).

Genomic analysis

CheckM (Parks et al., 2015) was used to assess the completeness and contamination in the DCMF genome. SPADE (Mori et al., 2019) was used to analyse repeat regions in the genomes, using default parameters.

The 81 full-length predicted trimethylamine (TMA) methyltransferase protein sequences were aligned with MAFFT v7.310 (Katoh et al., 2002) and a Maximum-Likelihood tree (1000 bootstraps) inferred by IQTree v1.6.1 using ModelFinder (Nguyen et al., 2015; Kalyaanamoorthy et al., 2017). Global pairwise percentage identities were calculated using GABLAM v2.28.2 (Davey, Shields \& Edwards, 2006) from an all-by-all BLAST 2.5.0+ blastp search (Camacho et al., 2009) .

Putative selenocystine-containing proteins were verified via multiple lines of evidence. The presence of a selenocysteine insertion sequence (SECIS) was confirmed in either the JGI annotation, or via bSECISearch (Zhang \& Gladyshev, 2005). Glycine/betaine/sarcosine reductase genes were checked for the presence of the conserved cysteine(s) present either before (CxxU in $\operatorname{grdA})$ (Kreimer \& Andreesen, 1995) or after (UxxCxxC in $\operatorname{grdBFH})$ the selenocysteine residue (Wagner et al., 1999). In order to determine the substrate specificity of these reductases, the predicted proteins encoding the two subunits of B component (GrdB/F/H and $\mathrm{GrdE} / \mathrm{G} / \mathrm{I}$ ) were aligned with known glycine, glycine betaine, and sarcosine reductase B components from Clostridium sticklandii, Peptoclostridium acidaminophilum, Peptoclostridium litorale, Sporomusa ovata An4, and Sporomusa ovata H1 with MUSCLE in UGENE v.1.32 (Okonechnikov et al., 2012) and an unrooted Maximum Likelihood tree (1000 bootstraps) was inferred by IQ-Tree v1.6.1 using ModelFinder (Nguyen et al., 2015; Kalyaanamoorthy et al., 2017).

\section{Results}

\section{Enrichment of DCMF in DFE}

Five 1\% transfers (T1 - T5) of the previously reported enrichment culture DCMD (Lee et al., 2012) were carried out. The initial three transfers produced methane in a molar ratio of 0.6 moles per mole of DCM (Figure 2A). Addition of BES to the culture medium in T4 caused methanogenesis to cease, and T5 could utilise DCM without the generation of methane in the absence of BES (Figure 2B). The absence of methanogenic populations was confirmed via archaeal specific PCR. While a clear band at $\sim 660$ bp was observed in a positive control and T3 
293 culture, there was no archaeal PCR product from the enrichment culture after the addition and 294 subsequent removal of BES. The non-methanogenic, DCM-fermenting enrichment culture was 295 henceforth called DFE.

296 T5 was then subject to two rounds of dilution to extinction. Community diversity was monitored 297 throughout these transfers by DGGE, which showed a trend towards purity, culminating in the 298 presence of a single band from the lowest active dilution series culture $\left(10^{-3}\right.$; Figure S1).

299 Sequencing of the primary band had the highest identity match to an uncultured Peptococcaceae, 300 henceforth referred to as "DCMF".

301 The shift away from the Dehalobacter population originally shown to be linked to DCM-

302 degradation (Lee et al., 2012), was confirmed with qPCR. The Dehalobacter sp. 16S rRNA gene 303 was below the limit of detection $\left(1.45 \times 10^{3}\right.$ copies $\left.\mathrm{ml}^{-1}\right)$ at all stages of growth in DFE cultures after the removal of methanogenic populations.

306

307

308

309

310

311

312

313

314

315

316

317

318

319

320

321

322

323

324

325

326

327

328

329

Genome assembly and annotation

Attempts were initially made to sequence the dominant, DCM-degrading organism using Illumina short read technology, which yielded 5,040,903 filtered read pairs for a total of $1,827,383,271 \mathrm{bp}$. However, the presence of the additional organisms in the DFE culture and lack of a reference genome hindered this approach. Instead, a pure PacBio long read strategy was used to assemble a full-length gap-free circular genome for DCMF Trimmed and filtered Illumina reads (average $242 \times$ coverage) were used for final, minor error correction. The final genome assembly had an average of $132 \times$ PacBio coverage ( $\min >50 \times$ ) and no regions of unusual read depth (Figure 3A). The genome was circularised at overlapping ends and every base was covered by long reads spanning at least $5 \mathrm{~kb} 5$ ' and 3' (Figure 3B). In addition to these assessments, CheckM evaluated the genome as $97 \%$ complete with a contamination rate of $2 \%$.

The DCMF genome is $6,441,270$ bp long and has a G+C content of $46.44 \%$ (JGI genome ID 2718217647; GenBank accession CP017634.1). JGI annotation initially revealed 5,801 predicted protein-coding genes. Manual curation of the 28 pairs of genes fragmented by the presence of the amino acids pyrrolysine and selenocysteine (encoded by in-frame UAG and UGA stop codons, respectively; Table S3) brought this total down to 5,773 protein coding genes (Table 1).

16S rRNA gene phylogeny

The DCMF genome contains four full-length 16S rRNA genes (JGI locus tags Ga0180325_11664, 11677, 113771, 114507; Table S5), which share $99.87 \%$ identity when aligned. Based on the consensus $16 \mathrm{~S}$ rRNA gene sequence, the closest relative to DCMF is $D$. formicoaceticum strain DMC ( $94 \%$ identity). This is closely followed by ' $\mathrm{Ca}$.

Dichloromethanomonas elyunquensis' strain RM, Dehalobacter restrictus strain PER-K23 and Desulfosporosinus acidiphilus strain SJ4 (all 89\% identity), and Desulfitobacterium dehalogenans strain ATCC 51507 (88\% identity) (Figure 4). The lowest taxonomic rank of SILVA classification was the family Peptococcaceae. 
330 From the NR contaminants, 17 16S rRNA sequences were identified by barrnap. Based on

331 SILVA classification, the 17 sequences were clustered into five distinct classifications (identified

332 here by their lowest classified taxonomic rank): Synergistaceae, Spirochaetaceae, Desulfovibrio,

333 Ignavibacteria, and Lentimicrobiaceae (Table S5). These were supported by clear clades within

334 the SILVA tree (Figure S2).

335 Phylogenetic analysis of the predicted proteome

336 Taxonomic analysis of the whole predicted DCMF proteome was inconclusive at the genus level

337 but strongly supported assignment within the order Clostridiales (Figure 5). The top-ranked

338 genus was Dehalobacterium (25.7\% proteins, bootstrap-weighted), supporting the 16S rRNA

339 gene phylogeny (Figure 4) with D. formicoaceticum as the closest known relative of DCMF. The top families were Peptococcaceae (39.3\%) and Clostridiaceae (11.2\%).

341 Whole-proteome TaxaMap analysis provides a good overview but is clearly influenced by the

342

343

344

345

346

347

348

349

350

351

352

353

354

355

356

357

358

359

360

361

362

363

364

365

366

367

368 availability of homologous sequences in the search databases and may also be disrupted by, for example, horizontal gene transfer. We therefore restricted analysis to a more robust set of eight house-keeping genes and 47 ribosomal proteins (Table S4). With the exception of one malate dehydrogenase (Ga0180325_112460) and SSU ribosomal protein S10P (Ga0180325_114571), all proteins support $D$. formicoaceticum as the closest known relative of DCMF and placement in the Peptococcaceae family. All 55 genes support placement in Clostridiales (Table S4). Multiple sequence alignments, phylogenetic trees and TaxaMap assignments for all proteins can be found in online supplementary material at: http://www.slimsuite.unsw.edu.au/research/dcmf/. The restricted housekeeping genes can be found at:

http://www.slimsuite.unsw.edu.au/research/dcmf/dcmf-hk.php.

\section{Genomic features of DCMF}

A number of metabolic pathways were identified in the DCMF genome (Table 1, Figure 6). The most prominent of these is the full set of genes for the Wood-Ljungdahl pathway (Figure 6, Table S6). No reductive dehalogenases were identified in the genome by any of the three independent annotation pipelines.

The genome also contains an abundance of methylamine methyltransferase genes (Table S6), including 82 copies of TMA methyltransferase, $m t t B$. There is a high diversity amongst the $m t t B$ genes, with an average amino acid sequence identity of only $30.3 \%$. Associated with the presence of these methyltransferases are all five genes necessary to synthesise and utilise pyrrolysine (Table S8), a non-canonical amino acid residue present in 23 of the 96 total methylamine methyltransferases in the genome. In a maximum likelihood phylogenetic tree contructed from the 81 full-length $m t t B$ genes, the pyrrolysine-containing copies tend to cluster together at the bottom of the tree (Figure S3). The pyrrolysine gene cluster in DCMF includes the dedicated tRNA ( $p y l T)$, tRNA synthetase ( $p y l S c$ and $p y l S n)$, and associated biosynthetic enzymes ( $p y l B C D)$ (reviewed in Krzycki, 2013).

The presence of all genes required for de novo corrinoid biosynthesis (Table S9) is pertinent both to certain Wood-Ljungdahl pathway proteins and the methylamine methyltransferases, which 
369 require a corrinoid cofactor to function (Burke \& Krzycki, 1997; Ferguson et al., 2000).

370 However the genes for methionine synthesis (metH and metE), required to form $\mathrm{S}$ -

371 adenosylmethionine, which is in turn used as a methyl donor during corrin ring formation (Deeg

372 et al., 1977), were not identified in the genome. DCMF may be using an alternative route for de

373 novo biosynthesis of this amino acid.

374 Additionally, five clusters of glycine/sarcosine/betaine reductase complex genes were found

375 (Figure 6, Table S6), indicating that DCMF may have a wider metabolic repertoire than its

376 obligate DCM-fermenting relatives. Four of these clusters include the thioredoxin reductase

$377(\operatorname{tr} x B)$ and thioredoxin I $(\operatorname{tr} x A)$ necessary for electron transfer to the reductase, and the genome

378 also encodes betaine/choline/carnitine transporter genes necessary to import these compounds

379 into the cell. Components $\mathrm{A}(\mathrm{grdA})$ and $\mathrm{B}(\mathrm{grdBE} / F G / H I)$ of the glycine/sarcosine/betaine

380 reductase complex contain an integral selenocysteine residue, as does the formate dehydrogenase

381 (Ga0180325_112876, 112877, 112878s80) encoded in the DCMF genome. Accordingly, the

382 organism contains the full complement of genes necessary for biosynthesis and incorporation of

383 the unusual amino acid selenocysteine ( $\operatorname{sel} A B D$, ser $S$; Table S7). All predicted selenocysteine-

384 containing proteins also contain the SECIS downstream of the UGA stop codon, necessary for

385 translating it as a selenocysteine residue instead.

\section{Discussion}

387 The shift from a Dehalobacter species to DCMF

388 The novel Peptococcaceae, DCMF, was enriched from a previously reported methanogenic

389 consortium, DCMD, where DCM was supplied as the sole energy source (Lee et al., 2012). That

390 consortium was dominated by a Dehalobacter species whose growth was linked to DCM

391 metabolism, producing acetate and methane. The Archaeal population was dominated by a

392 hydrogenotrophic methanogen from the genus Methanoculleus. Furthermore, Dehalobacter sp.

393 growth could be inhibited by addition of excess hydrogen. These two phenomena led to the

394 conclusion that hydrogen was a DCM fermentation product along with acetate, and that a

395 syntrophic association existed between Dehalobacter and Methanoculleus (Lee et al., 2012). In

396 the present study, inhibition of methanogens with BES likely led to increased hydrogen levels,

397 which inhibited the growth of the Dehalobacter sp. and enabled the hitherto unknown non-

398 hydrogenogenic DCMF to become the dominant DCM fermenter in the DFE culture.

399 The cohabiting bacteria in the DFE culture have persisted despite attempts to isolate DCMF.

400 These have been limited to serial transfers of dilution to extinction, due to the inability of the

401 organism to form colonies on agar plates or in semi-solid agar shakes. Nonetheless, this has lead

402 to a highly enriched culture, with community fingerprinting results showing only a single

403 lineage.

404 Optimisation for a high quality genome assembly from a mixed culture

405 Based on the 16S rRNA gene sequence retrieved from the DGGE community analysis, DCMF

406 appeared to be an organism with comparatively few cultured relatives. Thus, whole genome

Peer) reviewing PDF | (2019:05:37241:2:0:NEW 23 Aug 2019) 
407

408

409

410

411

412

413

414

415

416

417

418

419

420

421

422

423

424

425

426

427

428

429

430

431

432

433

434

435

436

437

438

439

440

441

442

443

444

445

sequencing was carried out in order to learn more about its role and function in the enrichment community. The lack of a reference genome and other organisms in the enrichment culture hindered attempts to assemble the genome from short read sequences only, making the long read capability of PacBio sequencing indispensible for this effort. Although long reads are prone to a higher proportion of sequencing errors than short reads, a series of checks were put in place to ensure that a high quality, uncontaminated genome assembly was obtained.

The use of SMRTSCAPE to predict the optimal HGAP settings allowed rapid comparison of various assembly parameters. By increasing the minimum correction coverage from $6 \times$ to $10 \times$, the total size of the assembly (including contaminant organism DNA) decreased from $\sim 16 \mathrm{Mb}$ to $\sim 8.8 \mathrm{Mb}$, while the size of the DCMF genome remained relatively stable around $6.4 \mathrm{Mb}$. Increasing the minimum correction coverage one step further to $11 \times$ resulted in a significant reduction of the DCMF genome to $1.9 \mathrm{Mb}$, indicating that much of the assembly was likely being lost to overzealous correction (Table S2).

The large size of the DCMF genome distinguishes it from the two other known DCM-fermenting bacteria, D. formicocaceticum and "Ca. Dichloromethanomonas elyunquensis" (Table 1). When assembling a genome de novo from a mixed culture, there is always the concern that stretches of other contaminating genomes will be mis-incorporated into the assembly. This likelihood was reduced by our assembly strategy of increasing stringency. The consistent sequencing coverage across the final genome (Figure 3) strongly indicates that there was no such mis-assembly. The CheckM contaminant rate of $2 \%$ further confirms that the large DCMF genome is not overinflated due to contamination. Analysis of repeated sequence motifs with SPADE showed that they comprise just $21,395 \mathrm{bp}(0.03 \%)$ of the total DCMF genome, which also rules this out as a source of the large genome size. JGI annotation predicted 5,773 protein coding genes, giving a gene density of approximately 0.9 genes per kilobase, which is consistent with normal bacterial gene density (Koonin \& Wolf, 2008).

Genome annotation quality and availability of data

Despite the numerous error limiting and quality control steps taken in this study, it is almost certain that some errors will remain in both the genome sequence and genome annotation. We have therefore provided rich supplementary data to enable rapid, detailed analysis of potential genes and proteins of interest. The DCMF genome is available for browsing via a public Web Apollo (Lee et al., 2013) genome browser, accessed via the supplementary data site:

http://www.slimsuite.unsw.edu.au/research/dcmf/. Results of three annotation pipelines (Prokka, JGI and NCBI) are available through the browser for direct comparison, along with mapped PacBio reads for assessing genomic sequence quality. A search tool has also been provided, enabling Exonerate (Slater \& Birney, 2005) or BLAST+ (Camacho et al., 2009) searches of cDNA, peptides or genomic DNA against the DCMF genome, with hits linking directly to the corresponding region of the Web Apollo genome browser. Furthermore, multiple sequence alignments and phylogenetic trees have been provided for every JGI- and NCBI- annotated protein, enabling rapid assessment of protein descriptions and completeness. The genome and all 
446 annotation, including multiple sequence alignments and phylogenetic trees have been provided

447 for every protein, are available to download from Open Science Foundation (Edwards et al., 448 2019).

449 Central carbon and energy metabolism in DCMF

450 The genome of DCMF suggests that it dechlorinates DCM via incorporation into the Wood451 Ljungdahl pathway in a similar manner to that suggested for the two other DCM-fermenting 452 bacteria, D. formicoaceticum and ' $C a$. Dichloromethanomonas elyunquensis' (Mägli, Messmer 453 \& Leisinger, 1998; Kleindienst et al., 2019). All genes for this pathway were identified within 454 the genome, as well as those linking acetyl CoA to pyruvate and central carbon metabolism 455 within the cell (Figure 6, Table S6). DCM catabolism via the Wood-Ljungdahl pathway would 456 result in the production of acetate, and possibly formate. The DCMF genome encodes a 457 cytoplasmic formate dehydrogenase (Ga0180325_112876, 112877, 112878s80) that could 458 theoretically oxidise the formate to $\mathrm{CO}_{2}$, however it remains unclear whether it is functional 459 within the cell. Mägli et al (1996) found that, despite the presence of formate dehydrogenase

460 461

462 463

464

465

466

467

468

469

470

471

472

473

474

475

476

477

478

479

480

481

482

483

484 activity in cell extracts, D. formicoaceticum could not further metabolise the formate it produced, and instead accumulated it with acetate in a 2:1 molar ratio.

The DCMF genome also encodes an $\mathrm{F}_{1} \mathrm{~F}_{\mathrm{O}}$-type ATPase (Figure 6; Table S6), suggesting that it could employ a chemiosmotic mechanism for energy conservation alongside substrate-level phosphorylation of DCM. D. formicoaceticum and 'Ca. Dichloromethanomonas elyunquensis' also encode this ATPase; and evidence for its activity has been detected in cell-free extracts of the former and via proteomics for the latter (Mägli, Messmer \& Leisinger, 1998; Chen et al., 2017; Kleindienst et al., 2019). DCMF may generate a proton- or sodium-motive force for this ATPase via the Rnf complex, an ion-motive ferredoxin-NAD oxidoreductase encoded in its genome (Figure 6; Table S6). The Rnf complex is of particular importance given the absence of any electron-bifurcating hydrogenases in the genome. Typically, it pumps ions out of the cell, catalyzing electron transfer from reduced ferredoxin to $\mathrm{NAD}^{+}$(Biegel, Schmidt \& Müller, 2009; Biegel \& Muller, 2010), while the ATPase uses the flow of ions back into the cytoplasm to convert ADP to ATP. However, these two transmembrane protein complexes can also act in reverse in order to balance the pool of reduced electron carriers within the cell (e.g. Lechtenfeld et al., 2018).

An abundance of methyltransferases may indicate key role in DCM and wider metabolism The protein responsible for the dechlorination of DCM remains elusive within DCM-fermenting bacteria. However, there is growing evidence that a novel methyltransferase is responsible for transforming DCM into 5,10-methylene-THF, which then enters the Wood-Ljungdahl pathway (Mägli, Messmer \& Leisinger, 1998; Chen et al., 2018; Kleindienst et al., 2019). DCMF encodes an abundance of predicted methyltransferase proteins in its genome, hinting at a key role in metabolism. There are 96 genes annotated as a component of a methylamine methyltransferase system. They comprise the methyltransferase I (MTI, the $m t \_B$ genes), which transfers a methyl group onto a substrate specific corrinoid protein (the $m t \_C$ genes), from which the

Peer] reviewing PDF | (2019:05:37241:2:0:NEW 23 Aug 2019) 
485

486

487

488

489

490

491

492

493

494

495

496

497

498

499

500

501

502

503

504

505

506

507

508

509

510

511

512

513

514

515

516

517

518

519

520

521

522

523

methyltransferase II (MTII, $m t b A$ ) transfers the methyl group to the final receiving compound (coenzyme M in methanogenic archaea or THF in acetogenic bacteria) (van der Meijden et al., 1983a; van der Meijden et al., 1983b; Sauer and Thauer, 1997; Sauer et al., 1997).

The majority (82) of the 96 assorted methylamine methyltransferase genes in DCMF are annotated as component B of a TMA methyltransferase $(m t t B)$. The remaining genes include diand monomethylamine methyltransferases $(m t b B$ and $m t m B$ ), the cognate corrinoid proteins for tri-, di- and monomethylamine ( $m t t C, m t b C, m t m C$ ), and the non-specific corrinoid protein methyltransferase ( $m t b A)$ (Table S6).

The high diversity amongst the TMA methyltransferases in DCMF (average pairwise amino acid sequence identity of $30.3 \%$ ) may indicate that these genes have diversified to accommodate cobalamin cofactors with various upper and lower ligands (Visser et al., 2016) and/or that they have more than one function within the cell, i.e., that they are in fact methyltransferases for a wider variety of substrates than merely methylamines. It has previously been shown that the chloromethane dehalogenase $\mathrm{CmuAB}$ is functionally similar to the monomethylamine methyltransferase MtaA (Studer et al., 2001). Moreover, four corrinoid-dependent methyltransferases were highly expressed in the proteome of DCM-fermenting ' $\mathrm{Ca}$.

Dichloromethanomonas elyunquensis'(Kleindienst et al., 2019), further indicating that the array of corrinoid-binding methyltransferases in DCMF, along with its complete corrinoid biosynthetic pathway, may be crucial to the metabolism of DCM.

Interestingly, 23 of the 96 methylamine methyltransferase genes contain a pyrrolysine residue, identifiable as an in-frame UAG (amber) stop codon. While the $m t t B$ gene is widespread amongst bacteria and archaea, most organisms do not encode the pyrrolysine residue (Srinivasan, 2002; Ticak et al., 2014). Indeed, the pylTSBCD gene cluster to synthesise and incorporate this non-canonical amino acid is limited to a small number of bacterial genera, including Desulfotomaculum, Desulfitobacterium, and Thermincola (Gaston, Jiang \& Krzycki, 2011) - all members of the Peptococcaceae family and close relatives of DCMF based on 16S rRNA phylogeny. D. formicoaceticum also encodes the $p y l$ genes, but " $\mathrm{Ca}$. Dichloromethanomonas elyunquensis" does not (Table 1).

It is worth noting that ' $\mathrm{Ca}$. Dichloromethanomonas elyunquensis' is unique among the three DCM-fermenting species in encoding reductive dehalogenase genes in its genome (Table 1). This finding, coupled with a recent dual carbon-chlorine isotopic analysis of the two previouslyreported DCM-fermenters (Chen et al., 2018), suggests that there are distinct DCM dechlorination mechanisms operating in these organisms. Based on the presence or absence of key pathways in the genome (Table 1) and phylogenetic analysis (Figure 4), DCMF appears to have more in common with $\mathrm{D}$. formicoaceticum than ' $\mathrm{Ca}$. Dichloromethanomonas elyunquensis'.

A genome-based model for amine metabolism in DCMF

DCMF, D. formicoaceticum, and ' $\mathrm{Ca}$. Dichloromethanomonas elyunquensis' have thus far only been cultured on DCM (Mägli, Wendt \& Leisinger, 1996; Kleindienst et al., 2017), but the larger 
524 genome of DCMF suggests that perhaps it has a wider substrate repertoire. As well as a potential 525 role in DCM dechlorination, the many TMA methyltransferase genes could in fact allow the 526 organism to use TMA as a substrate for growth. In a pathway akin to that outlined for Sporomusa 527 ovata An4 (Visser et al., 2016), TMA could be demethylated in a stepwise manner to

528 dimethylamine and monomethylamine (Figure 6). Each methyl group released would be capable 529 of providing six reducing equivalents when oxidised to $\mathrm{CO}_{2}$.

530 It has also been shown that a nonpyrrolysine $m t t B$ homolog within Desulfitobacterium hafniense 531 Y51 is in fact a glycine betaine methyltransferase, $m \operatorname{tg} B$ (Ticak et al., 2014). Glycine betaine 532 methyltransferase systems (including the MTI, MTII, and cognate corrinoid proteins outlined 533 above) have also been found in S. ovata An4 (Visser et al., 2016), S. ovata H1 DSM 2662, and 534 Acetobacterium woodii (Lechtenfeld et al., 2018). In S. ovata An4, the same genes were 535 proposed to further demethylate the resulting dimethylglycine to sarcosine (Visser et al., 2016).

536 When the glycine betaine MTI and MTII proteins from each of these organisms were searched 537 against the DCMF predicted proteome, a number of homologs were identified that may be used 538 for glycine betaine or dimethylglycine demethylation (Figure 6). In the MttB maximum 539 likelihood tree (Figure S3), the top five highest identity homologs to the MtgB proteins from 540 these species all clustered together in a distinct clade. Ga0180325_115483, annotated as a 541 trimethylamine methyltransferase, had the highest percentage amino acid identity (54-55\%) to 542 the MTI proteins from all five species. However, it sits isolated on the opposite strand of DNA to 543 all surrounding genes, thus making it an unlikely candidate. The second highest identity MTI 544 homolog to all bar one species was Ga0180325_114740 (54-55\% identity), which sits in a 545 neighbourhood with three methyl-THF methyltransferases (shown to act as an MTII in S. ovata 546 An4 (Visser et al., 2016)), two further TMA MTIs, a cognate corrinoid protein, and two 547 betaine/choline/carnitine transporter genes. This genetic neighbourhood contains all the requisite 548 components for the demethylation system, and is thus a good candidate for glycine betaine 549 metabolism in DCMF.

550 The glycine betaine MTII proteins of the Sporomusa and Acetobacterium species had a lower 551 percentage identity to the nearest homolog in DCMF (44-45\% to Ga018325_111809), and even 552 lower again to the second best hit (32-36\% to Ga018325_111232). This makes it difficult to 553 determine any definitive candidates for a glycine betaine or dimethylglycine MTII gene in 554 DCMF. Nonetheless, relevant MTIs and methyltransferase cognate corrinoid proteins once again 555 surround these lower identity homologs in the DCMF genome, making them further possible 556 candidates for glycine betaine and/or dimethylglycine demethylation in DCMF.

557 In addition to demethylation, glycine betaine and sarcosine can be reductively cleaved to form 558 tri- or monomethylamine, respectively, plus acetyl phosphate. The DCMF genome contains five 559 separate clusters with glycine/betaine/sarcosine reductases and the thioredoxin I and reductase 560 necessary for electron transfer to the reductase (Figure 6, Table S6). These reductase genes are 561 also present in D. formicoaceticum, but absent from " $\mathrm{Ca}$. Dichloromethanomonas elyunquensis" 562 (Table 1). The glycine/sarcosine/betaine reductase complex consists of three components: the 
563 selenocysteine-containing component A (GrdA); a two- or three-subunit component B, one of

564 which contains a selenocysteine residue ( $\mathrm{GrdBE} / \mathrm{FG} / \mathrm{HI})$; and component $\mathrm{C}$, which is post-

565 translationally combined into a single protein (GrdCD) (Andreesen, 2004). In order to determine

566 the substrate specificity of the component B genes in DCMF, they were aligned with known

567 component B genes from Clostridium sticklandii, Peptoclostridium acidaminophilum,

568 Peptoclostridium litorale, S. ovata An4, and S. ovata H1. Of the five B components in DCMF, 569 Ga0180325_114802 (GrdG) and Ga0180325_114803s (GrdF) are likely specific to sarcosine, as

570 they clustered with the sarcosine reductase genes from the two Sporomusa species, while

571 Ga0180325_11115251 (GrdI) and Ga0180325_115252s54 (GrdH) clustered with the glycine

572 betaine reductases (Figure S4). One of the remaining B components is likely a pseudogene

573 (Ga0180325_11855s), as it lacks the required UxxCxxC motif after the selenocysteine residue to

574 protect against accidental oxidation (Parther, 2003). The substrate-specificity of the remaining

575 two B components (Ga0180325_114453 and Ga0180325_114454s56; Ga0180325_114684 and

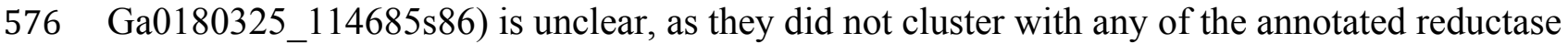

577 genes (Figure S4). The DCMF genome therefore suggests that this organism may be able to

578 metabolise methylated amine compounds via two different mechanisms, which could act in

579 concert: reductive cleavage and demethylation (Figure 6).

\section{Conclusions}

581 DCMF is an organism that demonstrates a relatively rare metabolism and harbours a large

582 genome. Both long and short read genome sequencing technology were used to compliment each

583 other and assemble a singular, circular chromosome for the organism, despite the low-level

584 presence of other bacteria in the enrichment culture. DCMF is the dominant organism in the

585 enrichment and likely sits within the Peptococcaceae family, although not within any known

586 genus. Its DCM-fermenting capabilities make it of interest to the bioremediation sector and the

587 genome contains clues to the as-yet undiscovered DCM dechlorinating enzyme, the identification

588 of which will be the subject of future work. The genome also suggests that DCMF can

589 metabolise a number of amine compounds, such as methylated amines, glycine betaine,

590 dimethylglycine, and sarcosine, though these capabilities remain to be tested. Extensive

591 supplementary data for the DCMF genome and annotation is available at

592 http://www.slimsuite.unsw.edu.au/research/dcmf/ and Open Science Foundation (Edwards et al., 593 2019).

\section{Acknowledgements}

595 We thank Dr Bat-Erdene Jugder (University of New South Wales) for his assistance with the 596 DNA extractions for PacBio sequencing and Dr Xabier Vázquez-Campos (University of New

597 South Wales) for assistance with data retrieval.

\section{References}

599 Adrian L, Manz W, Szewzyk U, Görisch H. 1998. Physiological characterization of a bacterial

600 consortium reductively dechlorinating 1,2,3- and 1,2,4-trichlorobenzene. Applied and 
601

602

603

604

605

606

607

608

609

610

611

612

613

614

615

616

617

618

619

620

621

622

623

624

625

626

627

628

629

630

631

632

633

634

635

636

637

638

639

640

641

\section{Environmental Microbiology 64:496-503.}

Agency for Toxic Substances and Disease Registry. 2000. Toxicological profile for methylene chloride. Atlanta, Georgia.

Andreesen JR. 2004. Glycine reductase mechanism. Current Opinion in Chemical Biology 8:454-461. DOI: 10.1016/j.cbpa.2004.08.002.

Biegel E, Muller V. 2010. Bacterial Na+-translocating ferredoxin:NAD+ oxidoreductase. Proceedings of the National Academy of Sciences 107:18138-18142. DOI: 10.1073/pnas.1010318107.

Biegel E, Schmidt S, Müller V. 2009. Genetic, immunological and biochemical evidence for a Rnf complex in the acetogen Acetobacterium woodii. Environmental Microbiology 11:1438-1443. DOI: 10.1111/j.1462-2920.2009.01871.x.

Burke SA, Krzycki JA. 1997. Reconstitution of monomethylamine:coenzyme M methyl transfer with a corrinoid protein and two methyltransferases purified from Methanosarcina barkeri. The Journal of Biological Chemistry 275:29053-60. DOI: 10.1074/jbc.M910218199.

Camacho C, Coulouris G, Avagyan V, Ma N, Papadopoulos J, Bealer K, Madden TL. 2009. BLAST+: architecture and applications. BMC Bioinformatics 10:1. DOI: Artn 421 \nDoi 10.1186/1471-2105-10-421.

Chaisson MJ, Tesler G. 2012. Mapping single molecule sequencing reads using basic local alignment with successive refinement (BLASR): application and theory. $B M C$ Bioinformatics 13:238. DOI: 10.1186/1471-2105-13-238.

Chen IMA, Chu K, Palaniappan K, Pillay M, Ratner A, Huang J, Huntemann M, Varghese N, White JR, Seshadri R, Smirnova T, Kirton E, Jungbluth SP, Woyke T, Eloe-Fadrosh EA, Ivanova NN, Kyrpides NC. 2019. IMG/M v.5.0: An integrated data management and comparative analysis system for microbial genomes and microbiomes. Nucleic Acids Research 47:D666-D677. DOI: 10.1093/nar/gky901.

Chen G, Murdoch RW, Mack EE, Seger ES, Löffler FE. 2017. Complete genome sequence of Dehalobacterium formicoaceticum strain DMC, a strictly anaerobic dichloromethanedegrading bacterium. Genome Announcements 5:18-19. DOI: https://doi .org/10.1128/genomeA.00897-17.

Chen G, Shouakar-Stash O, Phillips E, Justicia-Leon SD, Gilevska T, Sherwood Lollar B, Mack EE, Seger ES, Löffler FE. 2018. Dual carbon-chlorine isotope analysis indicates distinct anaerobic dichloromethane degradation pathways in two members of Peptococcaceae. Environmental Science \& Technology 52:8607-8616. DOI: 10.1021/acs.est.8b01583.

Chin C-S, Alexander DH, Marks P, Klammer AA, Drake J, Heiner C, Clum A, Copeland A, Huddleston J, Eichler EE, Turner SW, Korlach J. 2013. Nonhybrid, finished microbial genome assemblies from long-read SMRT sequencing data. Nature Methods 10:563-569. DOI: $10.1038 /$ nmeth.2474.

Cox MP, Peterson DA, Biggs PJ. 2010. SolexaQA: At-a-glance quality assessment of Illumina second-generation sequencing data. BMC Bioinformatics 11:485.

Davey NE, Shields DC, Edwards RJ. 2006. SLiMDisc: Short, linear motif discovery, correcting for common evolutionary descent. Nucleic Acids Research 34:3546-3554. DOI:

Peer) reviewing PDF | (2019:05:37241:2:0:NEW 23 Aug 2019) 
642

643

644

645

646

647

648

649

650

651

652

653

654

655

656

657

658

659

660

661

662

663

664

665

666

667

668

669

670

671

672

673

674

675

676

677

678

679

680

681

\subsection{3/nar/gk1486.}

Deeg R, Kriemler H-P, Bergmann K-H, Müller G. 1977. On cobyrinic acid biosynthesis. Novel methylated hydroporphyrins and their role in cobyrinic acid formation (author's transl). Hoppe-Seyler's Zeitschrift fur Physiologische Chemie 358:339-352.

Edwards RJ, Holland SI, Ertan H, Manefield M, Lee M. 2019. Holland et al (2019) Supplementary Data. DOI: 10.17605/OSF.IO/BK5MU.

Edwards RJ, Moran N, Devocelle M, Kiernan A, Meade G, Signac W, Foy M, Park SDE, Dunne E, Kenny D, Shields DC. 2007. Bioinformatic discovery of novel bioactive peptides. Nature Chemical Biology 3:108-112. DOI: 10.1038/nchembio854.

Ferguson DJJ, Gorlatova N, Grahame DA, Krzycki JA. 2000. Reconstitution of dimethylamine:coenzyme $\mathrm{M}$ methyl transfer with a discrete corrinoid protein and two methyltransferases purified from Methanosarcina barkeri. Journal of Biological Chemistry 275:29053-29060.

Freedman DL, Gossett JM. 1991. Biodegradation of dichloromethane and its utilization as a growth substrate under methanogenic conditions. Applied and Environmental Microbiology 57:113-2847-2857.

Freedman DL, Smith CR, Noguera DR. 1997. Dichloromethane biodegradation under nitratereducing conditions. Water Environment Research 69:115-122.

Fuchs G. 2011. Alternative pathways of carbon dioxide fixation: Insights into the early evolution of life? Annual Review of Microbiology 65:631-658. DOI: 10.1146/annurev-micro-090110102801.

Gaston MA, Jiang R, Krzycki JA. 2011. Functional context, biosynthesis, and genetic encoding of pyrrolysine. Current Opinion in Microbiology 14:342-349. DOI: 10.1016/j.mib.2011.04.001.

Gribble GW. 2009. Naturally Occurring Organohalogen Compounds: A Comprehensive Update. Springer Science \& Business Media. DOI: 10.1007/978-3-211-99323-1.

Han M, Zmasek C. 2009. phyloXML: XML for evolutionary biology and comparative genomics. BMC Bioinformatics 10:356. DOI: 10.1186/1471-2105-10-356.

Hossaini R, Chipperfield MP, Montzka SA, Leeson AA, Dhomse SS, Pyle JA. 2017. The increasing threat to stratospheric ozone from dichloromethane. Nature Communications 8:15962. DOI: $10.1038 /$ ncomms15962.

Kalyaanamoorthy S, Minh BQ, Wong TKF, von Haeseler A, Jermiin LS. 2017. ModelFinder: fast model selection for accurate phylogenetic estimates. Nature Methods 14:587-589. DOI: $10.1038 /$ nmeth.4285.

Katoh K, Misawa K, Kuma K, Miyata T. 2002. MAFFT: a novel method for rapid multiple sequence alignment based on fast Fourier transform. Nucleic Acids Research 30:3059-3066. DOI: $10.1093 /$ nar/gkf436.

Kleindienst S, Chourey K, Chen G, Murdoch RW, Higgins SA, Iyer R, Campagna SR, Mack EE, Seger ES, Hettich RL, Löffler FE. 2019. Proteogenomics reveals novel reductive dehalogenases and methyltrasnferases expressed during anaerobic dichloromethane

Peer] reviewing PDF | (2019:05:37241:2:0:NEW 23 Aug 2019) 
682

683

684

685

686

687

688

689

690

691

692

693

694

695

696

697

698

699

700

701

702

703

704

705

706

707

708

709

710

711

712

713

714

715

716

717

718

719

720

721

metabolism. Applied and Environmental Microbiology 85:1-16. DOI: 10.1128/aem.0276818.

Kleindienst S, Higgins SA, Tsementzi D, Chen G, Konstantinidis KT, Mack EE, Löffler FE. 2017. 'Candidatus Dichloromethanomonas elyunquensis' gen. nov., sp. nov., a dichloromethane-degrading anaerobe of the Peptococcaceae family. Systematic and Applied Microbiology 40:150-159. DOI: 10.1016/j.syapm.2016.12.001.

Kleindienst S, Higgins SA, Tsementzi D, Konstantinidis KT, Mack EE, Löffler FE. 2016. Draft genome sequence of a strictly anaerobic dichloromethane-degrading bacterium. Genome Announcements 4:e00037-16. DOI: 10.1128/genomeA.00037-16.

Koonin E V, Wolf YI. 2008. Genomics of bacteria and archaea: The emerging dynamic view of the prokaryotic world. Nucleic Acids Research 36:6688-6719. DOI: 10.1093/nar/gkn668.

Kreimer S, Andreesen JR. 1995. Glycine reductase of Clostridium litorale - Cloning, sequencing, and molecular analysis of the grdAB operon that contains two in-frame TGA codons for selenium incorporation. European Journal of Biochemistry 234:192-199.

Krzycki JA. 2013. The path of lysine to pyrrolysine. Current Opinion in Chemical Biology 17:619-625. DOI: 10.1016/j.cbpa.2013.06.023.

Kuraku S, Zmasek CM, Nishimura O, Katoh K. 2013. aLeaves facilitates on-demand exploration of metazoan gene family trees on MAFFT sequence alignment server with enhanced interactivity. Nucleic Acids Research 41:W22-W28. DOI: 10.1093/nar/gkt389.

Lechtenfeld M, Heine J, Sameith J, Kremp F, Müller V. 2018. Glycine betaine metabolism in the acetogenic bacterium Acetobacterium woodii. Environmental Microbiology 20:4512-4525. DOI: $10.1111 / 1462-2920.14389$.

Lee E, Helt GA, Reese JT, Munoz-Torres MC, Childers CP, Buels RM, Stein L, Holmes IH, Elsik CG, Lewis SE. 2013. Web Apollo: a web-based genomic annotation editing platform. Genome Biology 14:R93. DOI: 10.1186/gb-2013-14-8-r93.

Lee M, Low A, Zemb O, Koenig J, Michaelsen A, Manefield M. 2012. Complete chloroform dechlorination by organochlorine respiration and fermentation. Environmental Microbiology 14:883-894. DOI: 10.1111/j.1462-2920.2011.02656.x.

Leisinger T, Braus-Stromeyer SA. 1995. Bacterial growth with chlorinated methanes. Environmental Health Perspectives 103:33-36.

Li H. 2013. Aligning sequence reads, clone sequences and assembly contigs with BWA-MEM. BMC Bioinformatics 11:485.

Mägli A, Messmer M, Leisinger T. 1998. Metabolism of dichloromethane by the strict anaerobe Dehalobacterium formicoaceticum. Applied and Environmental Microbiology 64:646-650.

Mägli A, Wendt M, Leisinger T. 1996. Isolation and characterization of Dehalobacterium formicoaceticum gen. nov. sp. nov., a strictly anaerobic bacterium utilizing dichloromethane as source of carbon and energy. Archives of Microbiology 166:101-108. DOI: $10.1007 / \mathrm{s} 002030050362$.

Marshall KA, Pottenger LH. 2016. Chlorocarbons and Chlorohydrocarbons. In: Kirk-Othmer Encyclopedia of Chemical Technology. John Wiley \& Sons, Inc,. DOI: 
722

723

724

725

726

727

728

729

730

731

732

733

734

735

736

737

738

739

740

741

742

743

744

745

746

747

748

749

750

751

752

753

754

755

756

757

758

759

760

761

762

\subsection{2/0471238961.1921182218050504.a01.pub3.}

van der Meijden P, Heythuysen HJ, Pouwels A, Houwen F, van der Drift C, Vogels GD. 1983a. Methyltransferases involved in methanol conversion by Methanosarcina barkeri. Archives of Microbiology 134:238-242. DOI: 10.1007/BF00407765.

van der Meijden P, Jansen LPJM, Drift C, Vogels GD. 1983b. Involvement of corrinoids in the methylation of coenzyme M (2-mercaptoethanesulfonic acid) by methanol and enzymes from Methanosarcina barkeri. FEMS Microbiology Letters 19:247-251. DOI: 10.1111/j.1574-6968.1983.tb00551.x.

Melendez CR, Roman MD, Smith GB. 1993. Biodegradation of dichloromethane under denitrifying conditions by a waste water microbial community and by pure cultures of a Hyphomicrobium strain X. In: Abstracts of the 93rd General Meeting, American Society for Microbiology. Session 126, 381.

Mori H, Evans-Yamamoto D, Ishiguro S, Tomita M, Yachie N. 2019. Fast and global detection of periodic sequence repeats in large genomic resources. Nucleic Acids Research 47:e8. DOI: $10.1093 /$ nar/gky890.

Nguyen LT, Schmidt HA, Von Haeseler A, Minh BQ. 2015. IQ-TREE: A fast and effective stochastic algorithm for estimating maximum-likelihood phylogenies. Molecular Biology and Evolution 32:268-274. DOI: 10.1093/molbev/msu300.

Okonechnikov K, Golosova O, Fursov M, the UGENE team. 2012. Unipro UGENE: A unified bioinformatics toolkit. Bioinformatics 28:1166-1167. DOI: 10.1093/bioinformatics/bts091.

Parks DH, Imelfort M, Skennerton CT, Hugenholtz P, Tyson GW. 2015. CheckM: assessing the quality of microbial genomes recovered from isolates, single cells, and metagenomes. Genome research 25:1043-55. DOI: 10.1101/gr.186072.114.

Parther T. 2003. Die Peroxidase-Aktivität Selenocystein-haltiger Proteine des strikt anaeroben Bakteriums Eubacterium acidaminophilum [Translation: The peroxidase activity of selenocysteine-containing proteins of the strict anaerobic bacterium Eubacterium acidamino. Universität Halle.

Poehlein A, Schmidt S, Kaster A, Goenrich M, Vollmers J, Bertsch J, Schuchmann K, Voigt B, Hecker M, Daniel R, Thauer RK, Gottschalk G, Müller V. 2012. An ancient pathway combining carbon dioxide fixation with the generation and utilization of a sodium ion gradient for ATP synthesis. PLoS ONE 7:e33439. DOI: 10.1371/journal.pone.0033439.

Pruesse E, Peplies J, Glöckner FO. 2012. SINA: Accurate high-throughput multiple sequence alignment of ribosomal RNA genes. Bioinformatics 28:1823-1829. DOI: 10.1093/bioinformatics/bts252.

Ragsdale SW, Pierce E. 2008. Acetogenesis and the Wood-Ljungdahl pathway of CO2 fixation. Biochimica et Biophysica Acta 1784:1873-1898. DOI: 10.1016/j.bbapap.2008.08.012.

Sauer K, Harms U, Thauer RK. 1997. Methanol:coenzyme M methyltransferase from Methanosarcina barkeri: purification, properties and encoding genes of the corrinoid protein MT1. European Journal of Biochemistry 243:670-677. DOI: 10.1111/j.14321033.1997.t01-1-00670.x.

Sauer K, Thauer RK. 1997. Methanol:coenzyme M methyltransferase from Methanosarcina

Peer] reviewing PDF | (2019:05:37241:2:0:NEW 23 Aug 2019) 
763

764

765

766

767

768

769

770

771

772

773

774

775

776

777

778

779

780

781

782

783

784

785

786

787

788

789

790

791

792

793

794

795

796

797

798

799

800

801

802

barkeri: Zinc dependence and thermodynamics of the methanol:cob(I)alamin methyltransferase reaction. European Journal of Biochemistry 249:280-285.

Seemann T. 2014. Prokka: Rapid prokaryotic genome annotation. Bioinformatics 30:2068-2069. DOI: 10.1093/bioinformatics/btu153.

Sievers F, Higgins DG. 2017. Clustal Omega for making accurate alignments of many protein sequences. Protein Science 27:135-145. DOI: 10.1002/pro.3290.

Slater GSC, Birney E. 2005. Automated generation of heuristics for biological sequence comparison. BMC Bioinformatics 6:31. DOI: 10.1186/1471-2105-6-31.

Srinivasan G. 2002. Pyrrolysine encoded by UAG in archaea: Charging of a UAG-decoding specialized tRNA. Science 296:1459-1462. DOI: 10.1126/science.1069588.

Stromeyer SA, Winkelbauer W, Kohler H, Cook AM, Leisinger T. 1991. Dichloromethane utilized by an anaerobic mixed culture: acetogenesis and methanogenesis. Biodegradation 2:129-137. DOI: 10.1007/BF00114603.

Studer A, Stupperich E, Vuilleumier S, Leisinger T. 2001. Chloromethane:tetrahydrofolate methyl transfer by two proteins from Methylobacterium chloromethanicum strain CM4. European Journal of Biochemistry 268:2931-2938. DOI: 10.1046/j.14321327.2001.02182.x.

The UniProt Consortium. 2017. UniProt: the universal protein knowledgebase. Nucleic Acids Research 45:D158-D169. DOI: https://doi.org/10.1093/nar/gkw1099.

Ticak T, Kountz DJ, Girosky KE, Krzycki JA, Ferguson DJ. 2014. A nonpyrrolysine member of the widely distributed trimethylamine methyltransferase family is a glycine betaine methyltransferase. Proceedings of the National Academy of Sciences of the United States of America:E4668-E4676. DOI: 10.1073/pnas.1409642111.

U.S. National Library of Medicine. 2019.TOXMAP. Available at https://toxmap.nlm.nih.gov/toxmap/app/

Urakawa H, Martens-Habbena W, Stahl DA. 2010. High abundance of ammonia-oxidizing archaea in coastal waters, determined using a modified DNA extraction method. Applied and environmental microbiology 76:2129-2135. DOI: 10.1128/AEM.02692-09.

US EPA. 2001. Fact Sheet: Correcting the Henry's Law Constant for Soil Temperature.

Visser M, Pieterse MM, Pinkse MWH, Nijsse B, Verhaert PDEM, de Vos WM, Schaap PJ, Stams AJM. 2016. Unravelling the one-carbon metabolism of the acetogen Sporomusa strain An4 by genome and proteome analysis. Environmental Microbiology 18:2843-2855. DOI: $10.1111 / 1462-2920.12973$.

Wagner M, Sonntag D, Grimm R, Pich A, Eckerskorn C, Söhling B, Andreesen JR. 1999. Substrate-specific selenoprotein B of glycine reductase from Eubacterium acidaminophilum. European Journal of Biochemistry 260:38-49. DOI: 10.1046/j.14321327.1999.00107.x.

Walker BJ, Abeel T, Shea T, Priest M, Abouelliel A, Sakthikumar S, Cuomo CA, Zeng Q, Wortman J, Young SK, Earl AM. 2014. Pilon: An integrated tool for comprehensive microbial variant detection and genome assembly improvement. PLoS ONE 9:e112963.

Peer) reviewing PDF | (2019:05:37241:2:0:NEW 23 Aug 2019) 
803

804

805

806

807

808

809

810

811

812

813

814

815

816

817

818

819

820

821

822

823

824

825

826

827

828

829

830

831

832

833

834

835

836

837

838

839

DOI: 10.1371/journal.pone.0112963.

Wolin EA, Wolin MJ, Wolfe RS. 1963. Formation of methane by bacterial extracts. Journal of Biological Chemistry 238:2882-2886.

Zhang Y, Gladyshev VN. 2005. An algorithm for identification of bacterial selenocysteine insertion sequence elements and selenoprotein genes. Bioinformatics 21:2580-2589. DOI: 10.1093/bioinformatics/bti400.

\section{Table and Figure Legends}

\section{Table 1. Comparison of the genomes of DCM-fermenting bacteria.}

Figure 1. DCMF genome assembly and annotation pipeline.

Figure 2. The removal of the methanogenic population from the DCM dechlorinating culture. (A) The initial three transfers (T1 - T3) of DCMD produced methane (black circles) in a molar ratio of 0.6 moles per mole DCM. DCM is shown both as actual concentration over time (white squares) as well as the cumulative DCM consumed (black squares). (B) DCM continued to be consumed in the presence (grey squares, subculture T4) and absence (white squares, subculture T5) of 2-bromoethanosulfonate, which caused methane production to cease.

Figure 3. Average coverage depth and read length across the DCMF genome assembly. (A) PacBio read depth along the full DCMF chromosome. Horizontal lines mark median depth (132×), and gradations as 1/8 median depth. (B) Maximum PacBio read length (kb) spanning each base along the full DCMF chromosome. Horizontal lines mark median length (15.3 kb), and gradations as 1/8 median length. Colours indicate total read length (blue), longest 5' distance from base spanned by a single read (purple), and longest 3' distance from base spanned by a single read (green).

Figure 4. 16S rRNA gene phylogenetic tree of DCMF with closely related bacteria (94-87\% identity). The two other known DCM-fermenting bacteria are underlined. Numbers indicate percentage of branch support from 1000 bootstraps. The scale bar indicates an evolutionary distance of 0.01 amino acid substitutions per site. Sequence were aligned in MAFFT program v.7 and a neighbor-joining tree (1000 bootstraps) resampling a 200PAM/k $=2$ scoring matrix was inferred using Archaeopteryx, with minor manual curation.

\section{Figure 5. Bootstrap-weighted combined taxonomic assignments for the DCMF predicted} proteome based on TaxaMap processing of high-throughput phylogenetic analysis. Results are shown at five taxonomic levels: genus, family, order, class and phylum. The asterisk (*) indicates where low abundance and/or unknown Firmicutes taxa have been combined at the genus, family, order and class levels.

Figure 6. A genome-based metabolic model for DCM and amine catabolism in DCMF. The known substrate DCM is proposed to be transformed via the Wood-Ljungdahl pathway (left). The genome also suggests that DCMF may be able to utilize one or more of: tri-, di- and 
840 monomethylamine, glycine betaine, dimethylglycine, and sarcosine (right). Both sides of the

841 model are predicted to produce acetyl compounds that can be transformed into pyruvate, linking

842 them with the central carbon metabolism of the cell. Enzymes are written in italics. Gene loci for

843 each enzyme are listed in Table S6. Abbreviations: ACS, acetyl CoA synthase; BCCT,

844 betaine/choline/carnitine transporter; DCM, dichloromethane; DMA, dimethylamine; DMG,

845 dimethylglycine; $\left(\mathrm{CH}_{3}\right) \mathrm{CFSP}$, (methyl) $\mathrm{Co}(\mathrm{I})$ corrinoid iron-sulfur protein; $\mathrm{CH} \equiv \mathrm{THF}, 5,10$ -

846 methenyl-tetrahydrofolate; $\mathrm{CH}_{2}=\mathrm{THF}, 5,10$-methylene-tetrahydrofolate; $\mathrm{CH}_{3}$-THF, 5-methyl-

847 tetrahydrofolate; $\mathrm{CHO}-\mathrm{THF}$, formyl-tetrahydrofolate; $\mathrm{CODH}$, carbon monoxide dehydrogenase;

848 Fd, ferredoxin; FolD, bifunctional 5,10-methylene-tetrahydrofolate dehydrogenase/5,10-

849 methylene-tetrahydrofolate cyclohydrolase; MMA, monomethylamine; MT, methyltransferase;

850 MTR, 5,10-methylene-tetrahydrofolate reductase; ox, oxidized; PFL, pyruvate formate lyase;

851 PFOR, pyruvate-ferredoxin oxidoreductase; red, reduced; THF, tetrahydrofolate; TMA,

852 trimethylamine; $\mathrm{Tr}$, thioredoxin.

853 


\section{Table 1 (on next page)}

Comparison of the genomes of DCM-fermenting bacteria. 
1 Table 1. Comparison of the genomes of DCM-fermenting bacteria.

2

\begin{tabular}{|c|c|c|c|}
\hline & "DCMF" & $\begin{array}{l}\text { Dehalobacterium } \\
\text { formicoaceticum }\end{array}$ & $\begin{array}{c}\text { "Candidatus } \\
\text { Dichloromethanomonas } \\
\text { elyunquensis" }\end{array}$ \\
\hline Genome Accession & $\begin{array}{c}2718217647 \\
(\mathrm{JGI})\end{array}$ & $\begin{array}{l}\text { CP022121.1 } \\
\text { (Genbank) }\end{array}$ & $\begin{array}{l}\text { LNDB00000000.1 } \\
\text { (Genbank) }\end{array}$ \\
\hline Genome size (bp) & $6,441,270$ & $3,766,545$ & $2,076,422$ \\
\hline$G+C$ content $(\%)$ & 46.4 & 43.2 & 43.5 \\
\hline Contigs & 1 & 1 & 53 \\
\hline Protein-coding sequences & 5,773 & 3,935 & 2,323 \\
\hline $\begin{array}{l}\text { Metabolic pathways/genes } \\
\text { of interest }\end{array}$ & & & \\
\hline Wood-Ljungdahl pathway & + & + & + \\
\hline Reductive dehalogenases & - & - & + \\
\hline Cobalamin biosynthesis & + & + & - \\
\hline $\begin{array}{l}\text { Glycine/betaine/sarcosine } \\
\text { reductase complex }\end{array}$ & + & + & - \\
\hline $\begin{array}{l}\text { Methylamine } \\
\text { methyltransferases }\end{array}$ & + & + & + \\
\hline Pyrrolysine biosynthesis & + & + & - \\
\hline Reference & This study & $\begin{array}{l}\text { (Chen et al., } \\
\text { 2017) }\end{array}$ & $\begin{array}{l}\text { (Kleindienst et al., } \\
\text { 2016) }\end{array}$ \\
\hline
\end{tabular}


Figure 1

DCMF genome assembly and annotation pipeline. 


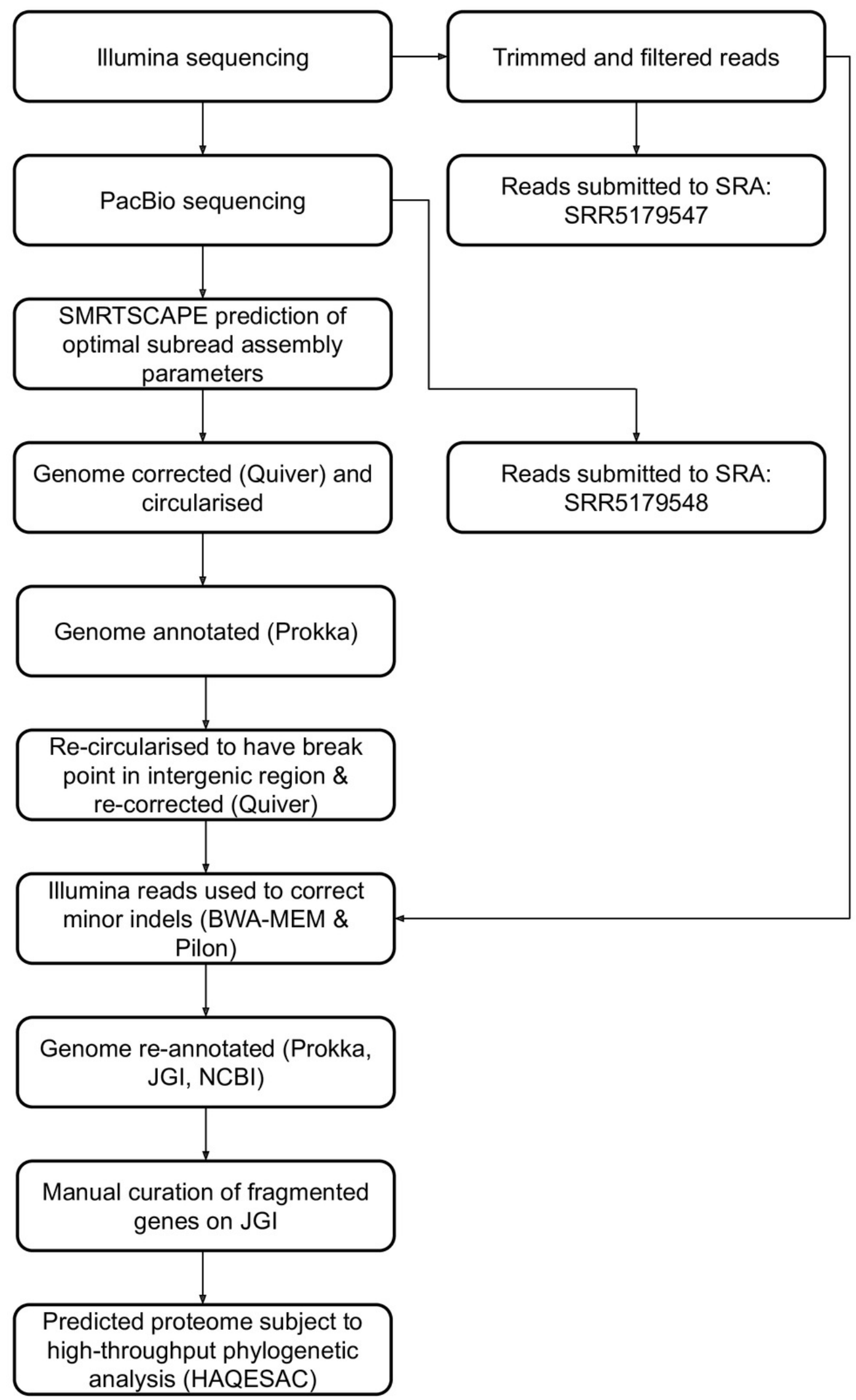




\section{Figure 2}

The removal of the methanogenic population from the DCM dechlorinating culture.

(A) The initial three transfers (T1 - T3) of DCMD produced methane (black circles) in a molar ratio of 0.6 moles per mole DCM. DCM is shown both as actual concentration over time (white squares) as well as the cumulative DCM consumed (black squares). (B) DCM continued to be consumed in the presence (grey squares, subculture T4) and absence (white squares, subculture T5) of 2-bromoethanosulfonate, which caused methane production to cease. 

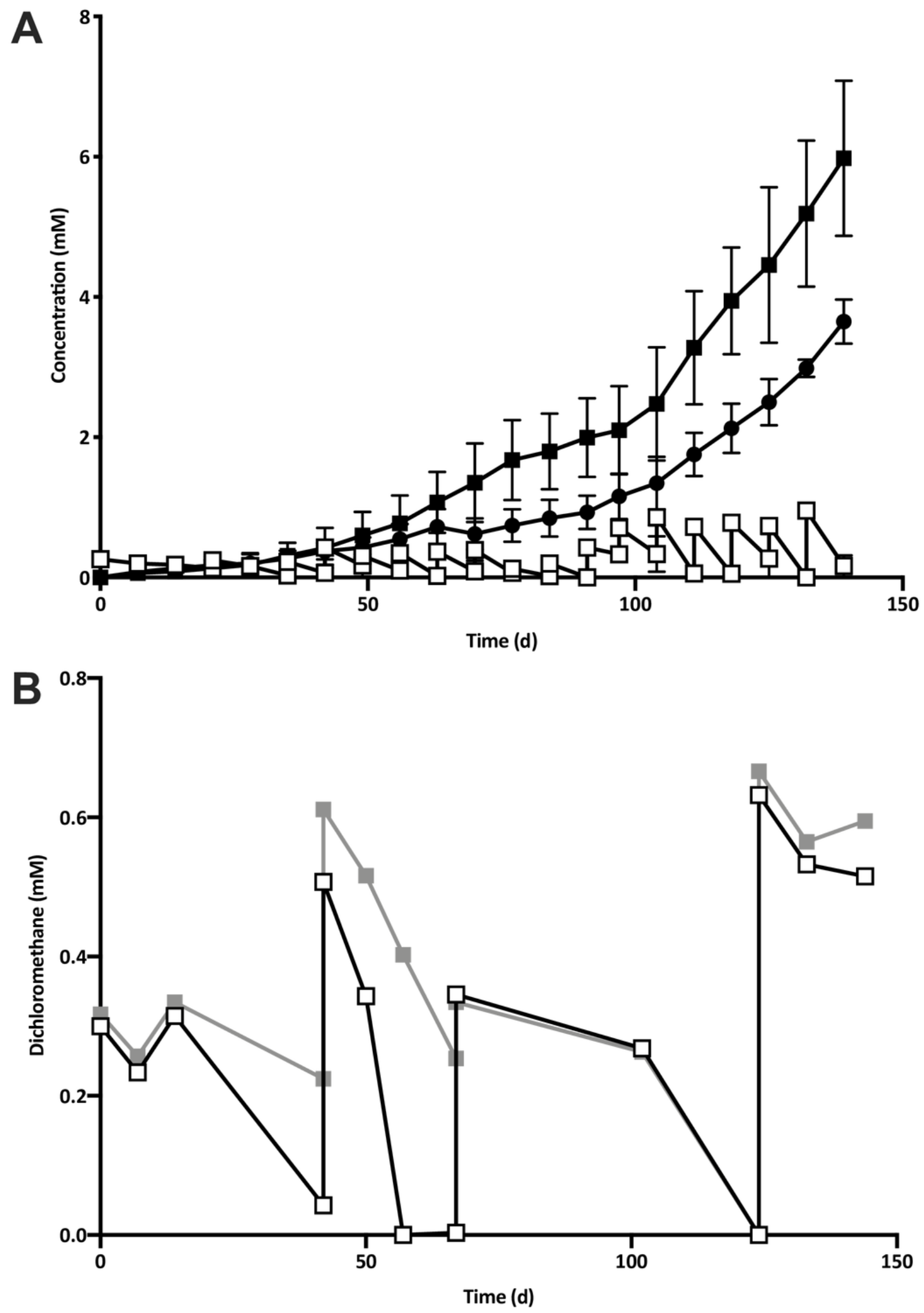


\section{Figure 3}

Average coverage depth and read length across the DCMF genome assembly.

(A) PacBio read depth along the full DCMF chromosome. Horizontal lines mark median depth (132x), and gradations as 1/8 median depth. (B) Maximum PacBio read length (kb) spanning each base along the full DCMF chromosome. Horizontal lines mark median length (15.3 kb), and gradations as 1/8 median length. Colours indicate total read length (blue), longest $5^{\prime}$ distance from base spanned by a single read (purple), and longest 3 ' distance from base spanned by a single read (green). 

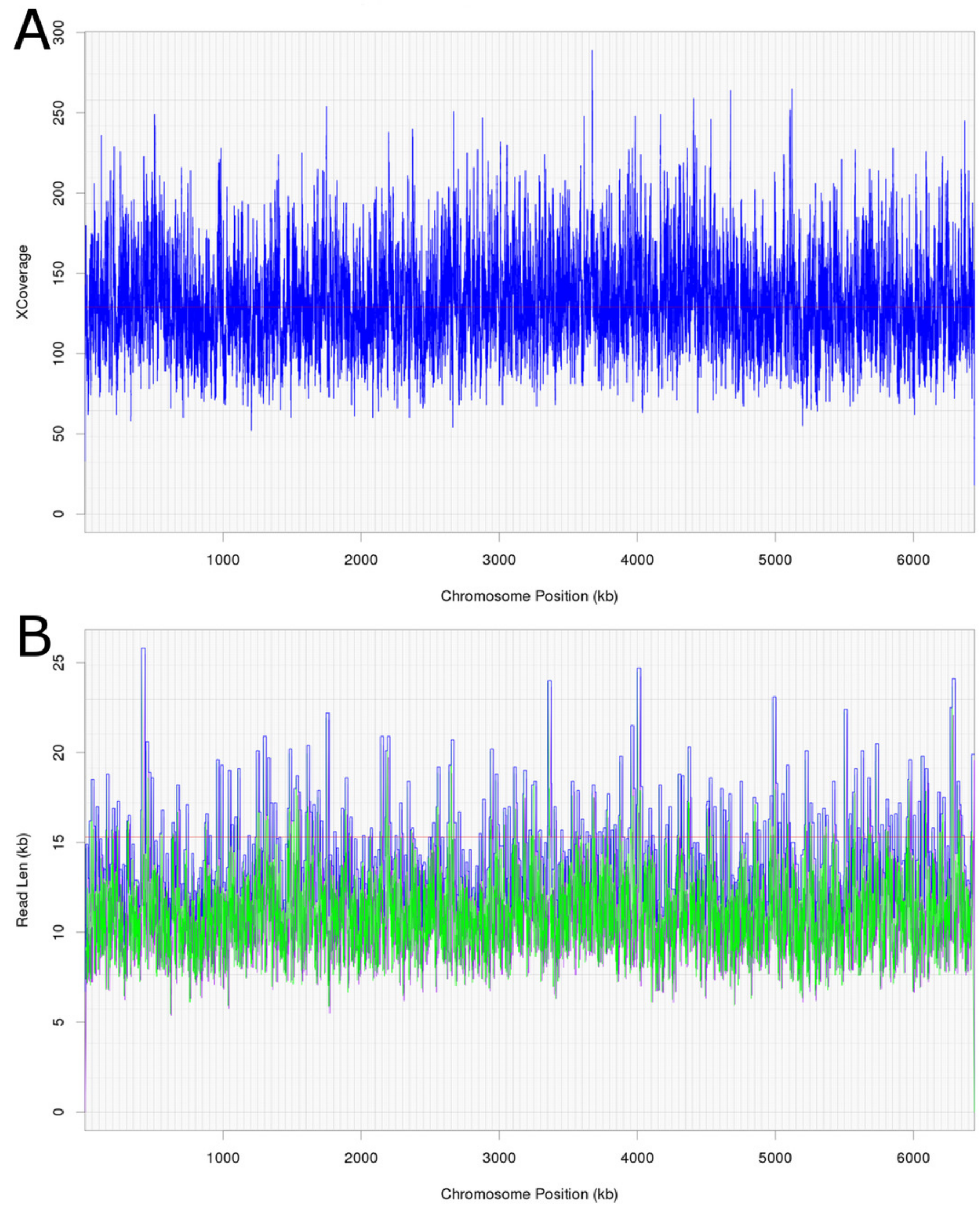


\section{Figure 4}

16S rRNA gene phylogenetic tree of DCMF with closely related bacteria (94-87\% identity).

Known DCM-fermenting bacteria are underlined . Numbers indicate percentage of branch support from 1000 bootstraps. The scale bar indicates an evolutionary distance of 0.01 amino acid substitutions per site. Sequence were aligned in MAFFT program v.7 and a neighbor-joining tree (1000 bootstraps) resampling a 200PAM/k =2 scoring matrix was inferred using Archaeopteryx, with manual curation.

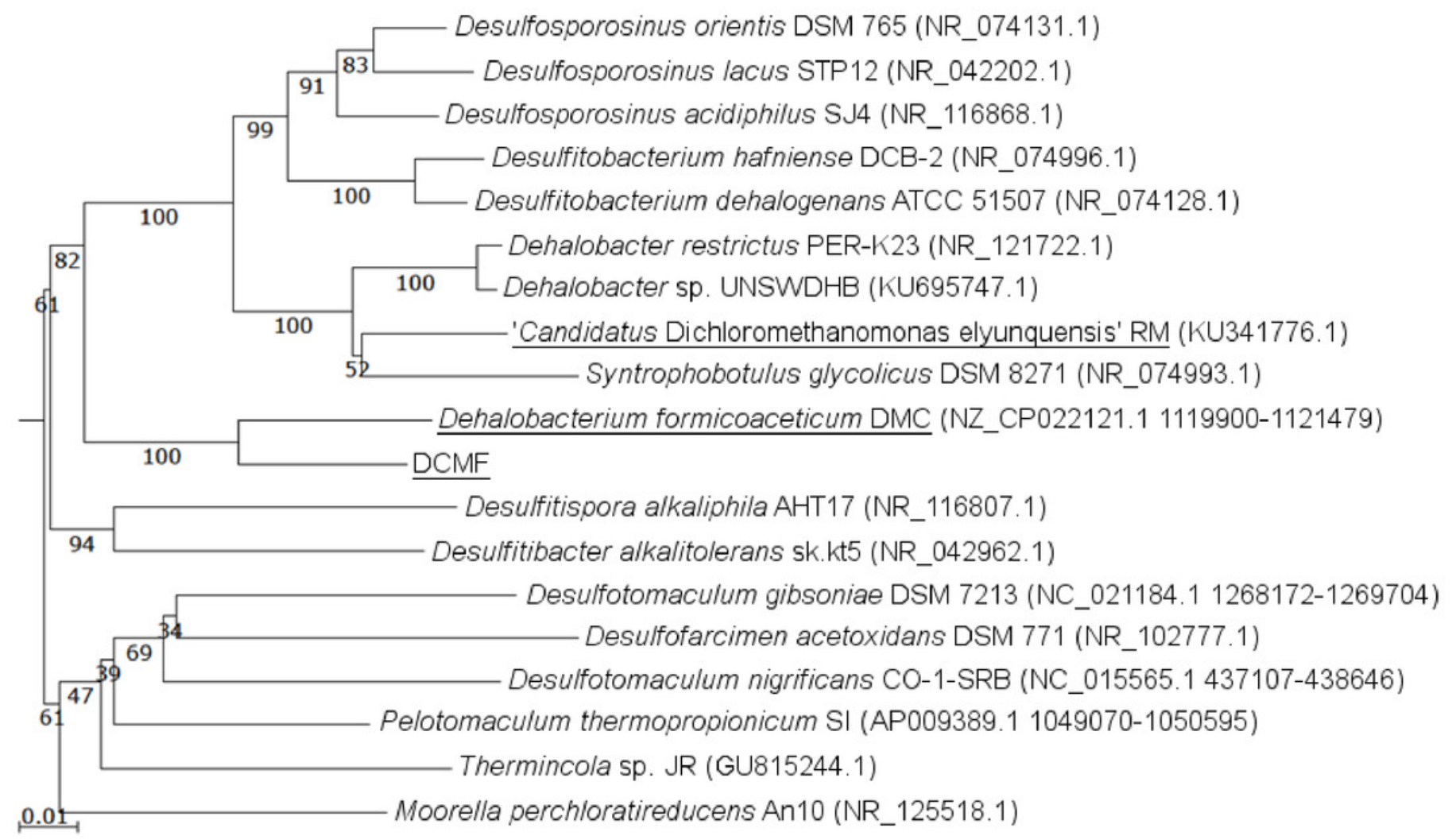




\section{Figure 5}

Bootstrap-weighted combined taxonomic assignments for the DCMF predicted proteome based on TaxaMap processing of high-throughput phylogenetic analysis.

Results are shown at five taxonomic levels: genus, family, order, class and phylum. The asterisk $(*)$ indicates where low abundance and/or unknown Firmicutes taxa have been combined at the genus, family, order and class levels.

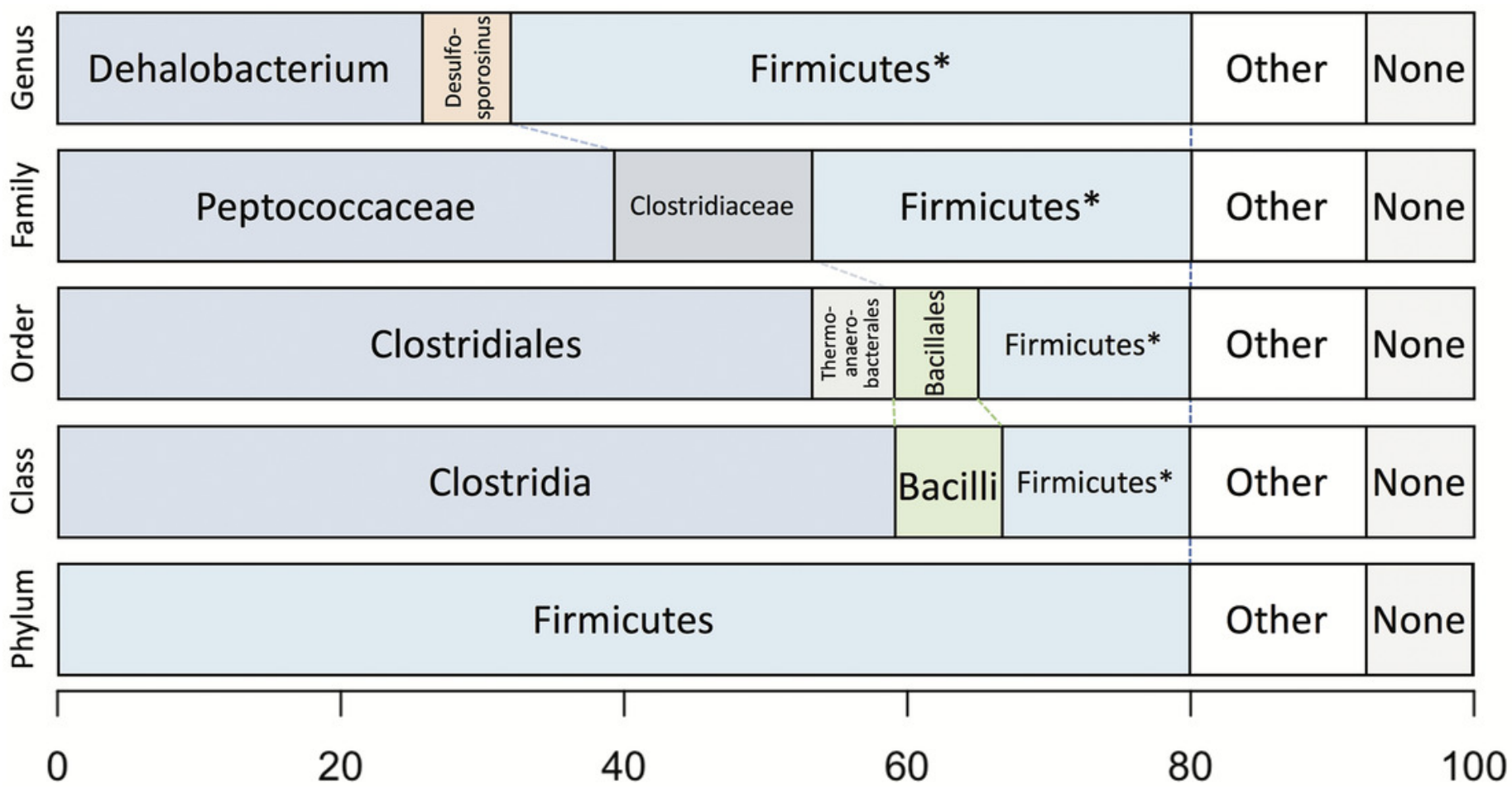




\section{Figure 6}

A genome-based metabolic model for DCM and amine catabolism in DCMF.

The known substrate DCM is proposed to be transformed via the Wood-Ljungdahl pathway (left). The genome also suggests that DCMF may be able to utilize one or more of: tri-, di- and monomethylamine, glycine betaine, dimethylglycine, and sarcosine (right). Both sides of the model are predicted to produce acetyl compounds that can be transformed into pyruvate, linking them with the central carbon metabolism of the cell. Enzymes are written in italics. Gene loci for each enzyme are listed in Table S6. Abbreviations: ACS, acetyl CoA synthase; BCCT, betaine/choline/carnitine transporter; DCM, dichloromethane; DMA, dimethylamine; DMG, dimethylglycine; $\left(\mathrm{CH}_{3}\right) \mathrm{CFSP}$, (methyl) $\mathrm{Co}(\mathrm{I})$ corrinoid iron-sulfur protein; $\mathrm{CH} \equiv \mathrm{THF}, 5,10$ methenyl-tetrahydrofolate; $\mathrm{CH}_{2}=\mathrm{THF}, 5,10$-methylene-tetrahydrofolate; $\mathrm{CH}_{3}$-THF, 5-methyltetrahydrofolate; $\mathrm{CHO}-\mathrm{THF}$, formyl-tetrahydrofolate; $\mathrm{CODH}$, carbon monoxide dehydrogenase; Fd, ferredoxin; FolD, bifunctional 5,10-methylene-tetrahydrofolate dehydrogenase/5,10-methylene-tetrahydrofolate cyclohydrolase; MMA, monomethylamine; MT, methyltransferase; MTR, 5,10-methylene-tetrahydrofolate reductase; ox, oxidized; PFL, pyruvate formate lyase; PFOR, pyruvate-ferredoxin oxidoreductase; red, reduced; THF, tetrahydrofolate; TMA, trimethylamine; $\mathrm{Tr}$, thioredoxin. 


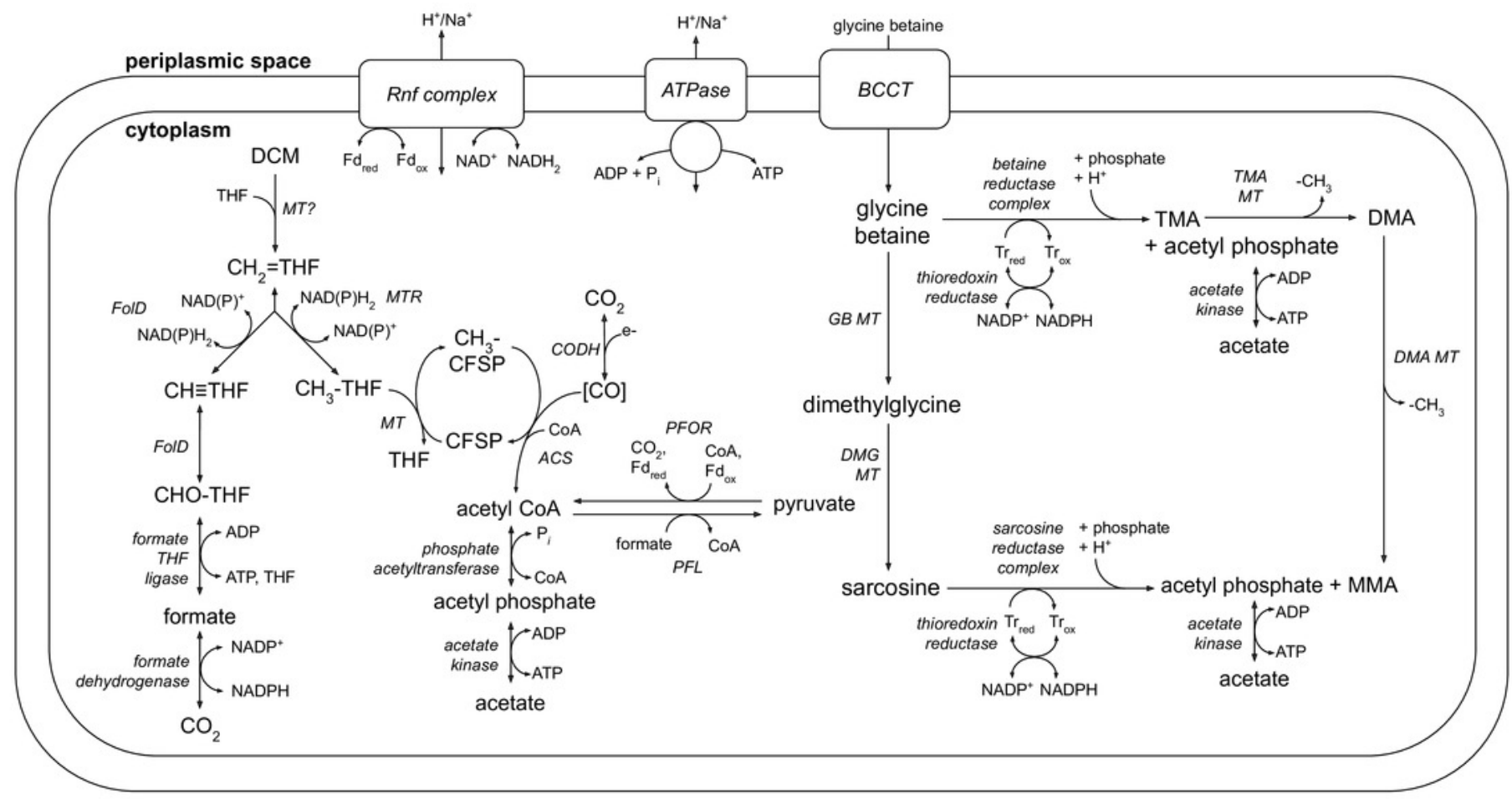

